\title{
FUN3D and USM3D Analysis of the Propulsion Aerodynamic Workshop 2018 S-duct Test Case
}

\author{
Melissa Carter,* Michael Bozeman, ${ }^{\dagger}$ and Jan-Reneé Carlson ${ }^{\ddagger}$ \\ NASA Langley Research Center, \\ Hampton, VA 23681, USA
}

\begin{abstract}
This work presents the results of Fun3D and USM3D analyses that were performed for the 4th AIAA Propulsion Aerodynamics Workshop (PAW). The PAW workshop is separated into three sections that focus on internal duct flows, nozzle flows and a special topic. This paper focuses on the internal duct flow section of PAW04 while an accompanying paper discusses the analyses performed for the nozzle portion. For the internal duct flow section, the PAW04 participants were provided with the two configurations consisting of an S-duct with and without aerodynamic interface plane (AIP) rake legs modeled. The participants were asked to perform a grid refinement study as well as a turbulence model study for the configuration with the rake legs. The analyses discussed here were performed on custom grids developed under the guidelines of the workshop. Additionally, the paper discusses the development and use of flow controllers for matching the desired flow characteristics. The results show that both solvers do well for predicting internal flow characteristics of the S-duct based on direct comparison with the experimental data. However, the CFD-to-CFD comparison proved to be more challenging due to the localized occurrence of supersonic flow near the rake legs when using the mass flow controller. A turbulence model study was performed to compare the two-equation SST model to the SA-QCR model. The results show that although the turbulence model does affect the solution, it makes a minimal impact on pressure recovery and inlet distortion intensity for this case. Suggestions for future workshops include gridding guidelines similar to those employed for the Drag Prediction Workshop series for the grid refinement study and a time accuracy study.
\end{abstract}

\section{Nomenclature}

$\begin{array}{ll}\mathrm{A} & =\text { area } \\ e & =\text { control error } \\ \gamma & =\text { ratio of specific heats } \\ \mathrm{k}_{\mathrm{p}}, \mathrm{k}_{\mathrm{i}}, \mathrm{k}_{\mathrm{d}} & =\text { PID-controller constants } \\ \mathrm{M} & =\text { Mach number } \\ \dot{m} & =\text { mass flow rate } \\ \mathrm{p} & =\text { pressure } \\ \mathrm{p}_{\mathrm{back}} & =\text { back pressure, } \mathrm{p} / \mathrm{p}_{\infty} \\ \mathrm{p}_{\infty} & =\text { freestream pressure } \\ \mathrm{P}_{T} & =\text { total pressure } \\ \mathrm{P}_{T, \infty} & =\text { freestream total pressure } \\ \mathrm{R} & =\text { gas constant } \\ \rho & =\text { density } \\ \mathrm{T} & =\text { temperature } \\ \mathrm{Tt} & =\text { total temperature } \\ \theta & =\text { angle (degree) } \\ \mathrm{AIP} & =\text { aerodynamic interface plane } \\ \mathrm{CFD} & =\text { computational fluid dynamics }\end{array}$

\footnotetext{
*Research Scientist, Configuration Aerodynamics Branch, Associate Fellow AIAA.

${ }^{\dagger}$ Research Scientist, Configuration Aerodynamics Branch, Member AIAA.

${ }^{\dagger}$ Research Scientist, Computational Aerosciences Branch, Associate Fellow AIAA.
} 


$\begin{array}{ll}\text { CFD2CFD } & =\text { CFD-to-CFD results comparison } \\ \text { CFD2EXP } & =\text { CFD-to-experimental results comparison } \\ \text { DPCP } & =\text { inlet distortion intensity } \\ \text { DPW } & =\text { drag prediction workshop } \\ 0 & =\text { output } \\ \text { PAW } & =\text { propulsion aerodynamics workshop } \\ \text { PID } & =\text { proportional-integral-derivative } \\ \text { PR } & =\text { pressure recovery } \\ \text { PRAV } & =\text { pressure ratio for a measurement ring } \\ \text { PRAVLOW } & =\text { pressure ratio below the average for a measurement ring } \\ \text { PV } & =\text { process variable } \\ \text { QCR } & =\text { quadratic constitutive relation } \\ \text { RANS } & =\text { Reynolds averaged Navier-Stokes } \\ \text { SA } & =\text { Spalart-Allmaras } \\ \text { SP } & =\text { setpoint } \\ \text { SST } & =\text { Menter's shear stress transport }\end{array}$

\section{Introduction}

$\mathrm{T}$ HE 4th Propulsion Aerodynamics Workshop (PAW04) was held at the 2018 AIAA Propulsion \& Energy Forum in Cincinnati, OH. Based on the success of other workshops such as the Drag Prediction Workshop (DPW), the PAW workshop was created to provide a forum for participants to work on common problems and share experiences. The PAW workshop series was motivated by the challenges associated with accurate predictions of steady and unsteady flows in inlets and nozzles with the goal of stimulating code improvements and enhancing the current state of the art. The workshop series is subdivided into sections focusing on the prediction of inlet and nozzle flows separately, as well as a special topic. For the inlet section of the workshop series, the main challenge that was identified is the accurate prediction of flow separation that occurs due to the offset between the inlet and engine faces resulting from the desire to make aircraft smaller and more compact [1]. For nozzles on the other hand, the challenge is the accurate prediction of jet flows over a range of operating conditions with and without integration effects. These problems have proved particularly challenging for Reynolds Averaged Navier-Stokes (RANS) solvers. This paper will focus on NASA Langley's participation in the inlet section of PAW04 while an accompanying paper will discuss the work performed for the nozzle section [2]. NASA Langley did not participate in the special topic portion of the workshop.

The internal flow test cases provided for the PAW04 participants consisted of an S-duct with and without the aerodynamic interface plane (AIP) rake legs modeled. The participants were asked to perform a grid refinement study as well as a turbulence model study for the configuration with the rake legs. A common set of grids were supplied to the participants consisting of coarse, medium, and fine. These grids were to be used to perform a grid refinement study to identify the necessary grid refinement for the remaining analyses. Alternatively, the participants were given the option of generating custom grids within provided guidelines. Upon completion of the grid refinement study, the selected grid refinement was to be used to perform a turbulence model study. The analysis of the grids were to be conducted in two different ways. The CFD-to-CFD result comparison, CFD2CFD, targeted the integrated mass flow at the exit of the computational model, where the average of the mass flow at the exit plane was to match the specified experimental value. The CFD-to-experiment result comparison, CFD2EXP, had the CFD matching the experimental conditions at the AIP by area-averaging the results at the location of the 40-probe rake.

For the work discussed here, the option of generating a custom set of grids was exercised. The simulations were performed using the NASA Langley developed USM3D and FUN3D flow solvers. This paper will discuss the grid generation that was performed, provide a description of the problem setup, the creation and use of flow controller for matching the desired flow characteristics, and finally show the results of the analyses performed. Additionally, some recommendations are provided for future PAW workshops based on the experiences of PAW04. 


\section{S-duct Test Case}

\section{A. Geometry}

The S-duct computational geometry was composed of a bell mouth contraction section, an adapter bridging between the bell mouth and the S-duct, the S-duct, the AIP, and a five diameter extension. Figure 1, provided by the PAW committee, shows the components and their relative sizes to each other. The AIP consists of a standard 40-probe pressure rake. The geometry used in PAW03 for the rake was incorrect and PAW04 included the correct geometry, which increased the rake legs area by approximately 50\%. The legs of the AIP were at 45-degree increments starting at zero degrees. The radii of the probe rings are shown in Table 1. The modeling of the the individual probes was not performed in this study, though it was an option for participants of the workshop.

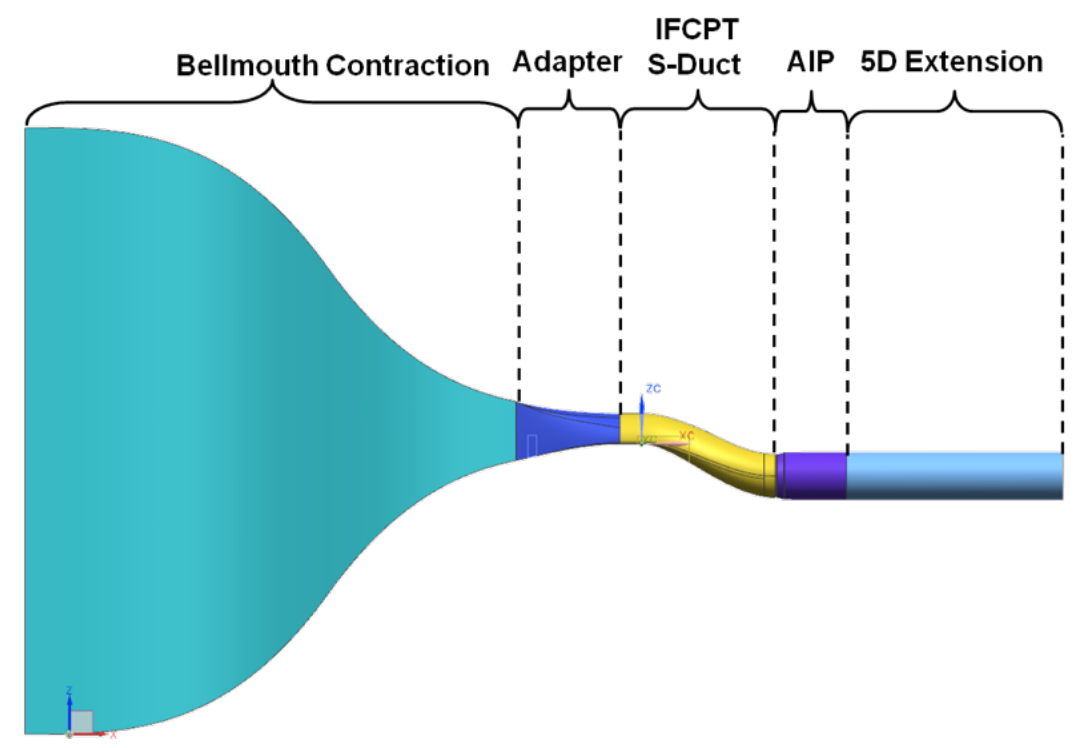

Fig. 1 S-duct configuration.

Table 1 Total pressure probe ring definition.

\begin{tabular}{cc}
\hline Ring & Radius [in] \\
\hline 1 & 0.791 \\
2 & 1.369 \\
3 & 1.768 \\
4 & 2.092 \\
5 & 2.372 \\
\hline
\end{tabular}

\section{B. Boundary Conditions and Grids}

The workshop defined that the inflow boundary condition at the entrance of the bellmouth should be set to ambient conditions. Additionally, the mass flow should be the specified condition at the downstream exit. The solid surfaces were modeled as viscous, no-slip walls. A series of grids were provided by the workshop both with and without the rake legs. Additional information about the grids used in this study can be found in Section III. C. 


\section{Prescribed Flow Conditions}

The goal of the workshop was for the computational methods to run at the same condition as the experiment. The measurements, as listed in Table 2, were all taken at the AIP. The Mach number and mass flow rate were determined from the isentropic flow equations using static and total pressure measurements at the AIP.

Table 2 Test conditions.

\begin{tabular}{rcccc}
\hline Parameter & $\begin{array}{c}\text { Mach } \\
\text { at AIP }\end{array}$ & $\begin{array}{c}\text { Total Pressure } \\
\left(\mathrm{lbf} / \mathrm{in}^{2}\right)\end{array}$ & $\begin{array}{c}\text { Total Temperature } \\
(\mathrm{R})\end{array}$ & $\begin{array}{c}\text { Mass Flow } \\
(\mathrm{lbm} / \mathrm{s})\end{array}$ \\
\hline Value & 0.6294 & 14.396 & 529.11 & 5.532 \\
\hline
\end{tabular}

\section{Inlet Pressure Recovery $(P R)$}

An inlet's performance is characterized by the inlet total pressure recovery. This is a measure of the amount of flow that matches freestream flow conditions. The calculation of total pressure recovery $\left(P R_{\text {avg }}\right)$ is shown in Eqn. 1 where $P_{T}$ is the total pressure measured by the pressure probe and $P_{T, \infty}$ is the freestream total pressure.

$$
P R_{\text {avg }}=\frac{1}{N} \sum_{i=1}^{N} P R_{i}
$$

where

$$
P R=\frac{P_{T}}{P_{T, \infty}}
$$
[3].

The theoretical limit of pressure recovery is 1.0. Additionally, the value obtained from the experiment was 0.9784

\section{E. Inlet Distortion Intensity ( $D P C P)$}

Another method of characterizing inlet performance is the distortion intensity (commonly abbreviated as DPCP). The basic concept for calculating the distortion intensity is depicted in Fig. 2 where the distortion intensity is calculated as the area identified in gray divided by the angular extent of the area (a detailed description of these calculations can be found in the SAE Report AIR1419) [4]. Note that the area indicated in gray is defined by the measurement locations that

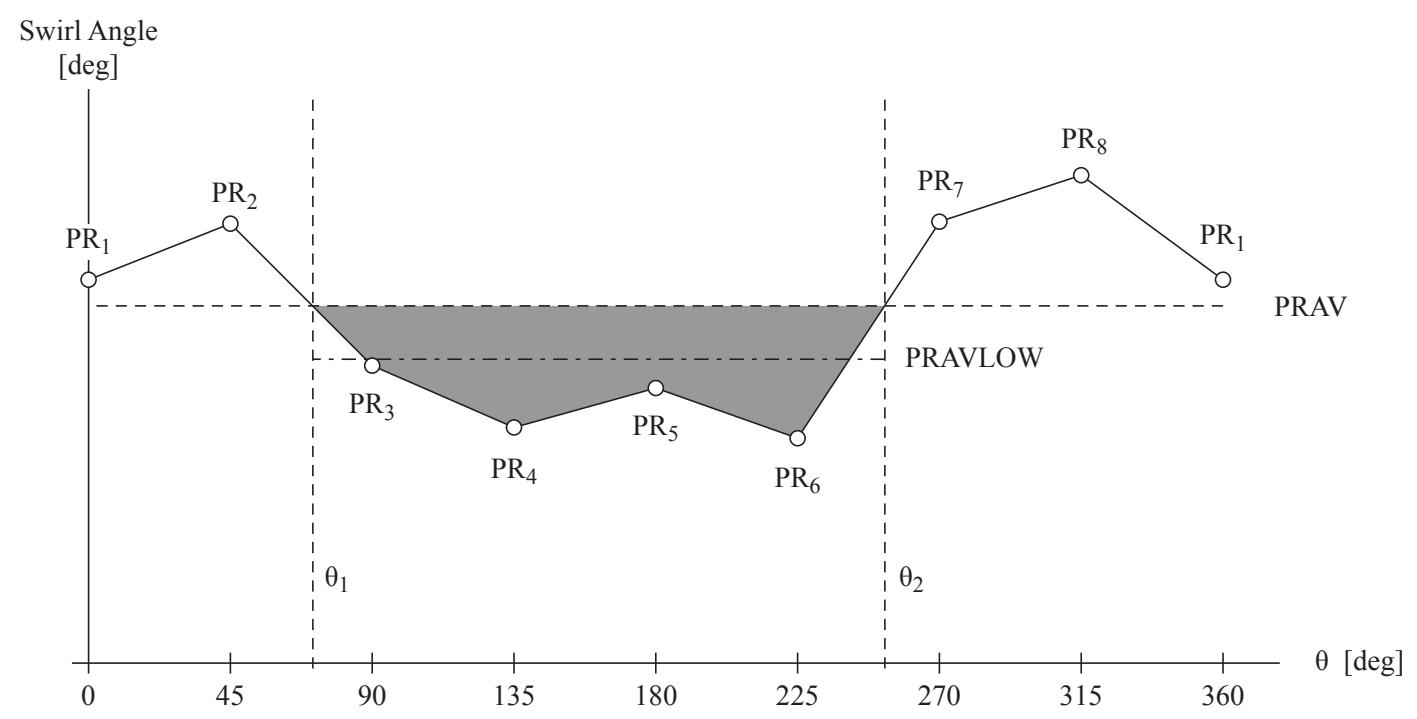

Fig. 2 Example dataset and nomenclature used for $D P C P$ calculations (adapted from SAE Report AIR1419[4]). 
have a pressure ratio (Eqn. 2) below the average ring pressure ratio. The average total pressure ratio for a measurement ring $(P R A V)$ is calculated in Eqn. 3, where the integral is computed discretely due to the finite number of measurement locations.

$$
P R A V=\frac{1}{360^{\circ}} \int_{0^{\circ}}^{360^{\circ}}(P R) d \theta
$$

Using the nomenclature defined in Fig. 2, PRAVLOW is calculated as

$$
P R A V L O W=\frac{1}{\theta} \int_{\theta_{1}}^{\theta_{2}}(P R) d \theta
$$

where the integral is again computed discretely due to the finite number of measurement locations. The angular extent of the area, $\theta$, is calculated as pressure ratio

$$
\theta=\theta_{2}-\theta_{1}
$$

$D P C P$ for a given ring (ring number represented as $i$ ) is calculated by normalizing $P R A V L O W$ by $P R A V$ (Eqn. 6).

$$
D_{P C P}=\frac{P R A V i-P R A V L O W i}{P R A V i}
$$

The maximum $D P C P$ is defined as the maximum $D P C P_{i}$ of all the rings (Eqn. 7).

$$
D P C P_{\max }=\max \left(D P C P_{i}\right)
$$

The total $D P C P$ for a measurement plane is calculated by taking the mean of the $D P C P$ ring values (Eqn. 8) where $R$ is the number of measurement rings.

$$
D P C P_{a v g}=\frac{1}{R} \sum_{i=1}^{R} D P C P_{i}
$$

The theoretical limit for DPCP is 0.0, while the value obtained experimentally for this case was 0.0098 [3].

\section{Computational Methods}

\section{A. USM3D}

\section{Code description}

The USM3D code [5] is a cell-centered, finite-volume Navier-Stokes flow solver that uses multiple flux splitting schemes including Roe's flux-difference splitting scheme [6] to compute inviscid flux quantities across the faces of the tetrahedral cells. Several options for turbulent closure are available: the one-equation Spalart-Allmaras (SA) model [7] with and without the QCR correction [8] and several two-equation models, including Menter's Shear Stress Transport (SST) model [9]. Throughout the study, the minmod limiter was used in order to keep the flow stable and provide consistent results. For the majority of the flow solution cases conducted in this study, the SST turbulence model was used.

\section{Boundary Conditions}

The tunnel inflow plane was modeled using the jet exit boundary condition, which is becoming standard when modeling a wind tunnel using USM3D. This boundary condition works by enforcing the total pressure and total temperature provided by the user to the code. The exit of the wind tunnel used a user-specified static pressure boundary condition.

\section{External Controller}

While FUN3D has a built in controller that is described later in this paper, USM3D currently requires an external program. This program can work in two ways. For the CFD to experimental cases the program was used in the following manner: 1) an initial back pressure is set by the user, 2) the 40 probe location points are specified to the code, which writes out the fundamental variables at each time step, 3) USM3D is run until convergence, 4) the Mach number is 
calculated from the last point of the probe data and a new back pressure is calculated based on the target Mach number, and 5) the code is run for 2000 iterations after which the Mach number is calculated again and the back pressure is altered as needed. For the medium grid, it took approximately 30 iterations to obtain the desired Mach number.

For the CFD-to-CFD comparisons, a preprocessor was run to mark the cells on the exit plane grid patch. Instead of having the code write data after each iteration, the script would read the values at the marked cells from the restart file after each successful run and compute the average Mach number. The back pressure would then be altered in the input file as necessary.

\section{B. Fun3D}

\section{Code description}

Fun3D is an unstructured three-dimensional, implicit, Navier-Stokes code. Roe's flux difference splitting [10] is used for the calculation of the inviscid terms. The method for calculation of the Jacobian was the flux function of van Leer [11]. Solutions computed with the Spalart-Almaras turbulence model [7] used the rotational correction [12] and the nonlinear stress QCR model [13], (SA-RC-QCR2000), and were run in both steady-state and time-accurate modes. Flux limiters were not used in any of the simulations, except where explicitly stated. Other details regarding Fun3D can be found in the manual [14], as well as in the extensive bibliography that is accessible at the Fun3D website [15].

\section{Boundary conditions and controller system}

This section discusses the determination of the inflow and outflow boundary conditions for the S-Duct simulations. The typical practice for modeling the inflow in an internal flow simulation is to specify the total pressure and total temperature [16-18]. At the exit, setting the static pressure, also referred to as the back pressure, is the most commonly used boundary condition. The total conditions at the inflow were determined from the isentropic equations using the Mach number desired at the minimum area in the duct. The back pressure was then adjusted using the boundary condition controller system. Additional implementation and theory details on Fun3D boundary conditions can be found in Carlson [18].

The boundary condition controller system consists of several parts: data acquisition, analyses of the data, processing by the controller logic and subsequent updating of the boundary condition value. The proportional-integral-derivative (PID) controller equations are general but the data sampling and condition analysis equations are specific to the simulation. The controller options for the driving the back pressure are currently 1-, 2- and 3-point controllers for subsonic wind tunnel simulations; total mass flow using the outflow boundary; total mass flow or Mach number using instrumentation at some location.

The analysis equations for the mass flow rate and Mach number controllers used in this study are shown in Eqs. 9-11, where $\mathrm{N}_{\text {Static }}$ and $\mathrm{N}_{\text {Total }}$ are the total number of static and total pressure measurement points, respectively. In these cases, Fun3D samples 8 static ports and the 40 total pressure probes in the rake at every iteration to calculate an average static pressure and temperature and an average total pressure and temperature at the location of the AIP. The averages are used to calculate new values for the Mach number at the AIP and the mass flow rate, which are used to update the controller output accordingly.

$$
\begin{gathered}
\overline{\mathrm{p}}=\sum_{n=1}^{\mathrm{N}_{\text {Static }}} \mathrm{p}, \quad \overline{\mathrm{T}}=\sum_{n=1}^{\mathrm{N}_{\text {Static }}} \mathrm{T}, \quad \overline{\mathrm{P}}_{\mathrm{T}}=\sum_{n=1}^{\mathrm{N}_{\text {Total }}} \mathrm{P}_{\mathrm{T}}, \quad \overline{\mathrm{T}}_{\mathrm{t}}=\sum_{n=1}^{\mathrm{N}_{\text {Total }}} \mathrm{T}_{\mathrm{t}} \\
\mathrm{M}_{\mathrm{AIP}}=\left[\frac{2}{\gamma-1}\left(\frac{\overline{\mathrm{P}}_{\mathrm{T}}}{\overline{\mathrm{p}}}\right)^{\left.\left(\frac{\gamma-1}{\gamma-1}\right)\right]^{\frac{1}{2}}}\right. \\
\dot{\mathrm{m}}=\frac{\overline{\mathrm{P}}_{\mathrm{T}}}{\sqrt{R \overline{\mathrm{T}}_{\mathrm{t}} / \gamma}} \mathrm{A} \mathrm{M}_{\mathrm{AIP}}\left(1+\frac{\gamma-1}{2} \mathrm{M}_{\mathrm{AIP}}^{2}\right)^{\frac{1}{2} \frac{\gamma+1}{1-\gamma}}
\end{gathered}
$$


The system uses a PID type controller,

$$
O=\mathrm{k}_{\mathrm{p}} \mathrm{e}+\mathrm{k}_{\mathrm{i}} \int_{0}^{t} \mathrm{e}(\tau) d \tau+\mathrm{k}_{\mathrm{d}} \frac{d \mathrm{e}}{d t}, \quad \mathrm{e}=\mathrm{SP}-\mathrm{PV},
$$

where the control signal is the sum of three terms: the proportional term as a function of the control error (e), the integral of the error, and the derivative of the error. The controller can be driven by several different variables: Mach number, mass flow rate, dynamic pressure, or Reynolds number. The setpoint (SP) is the target value of the parameter being used to drive the controller, e.g., Mach number at the AIP or mass flow rate. The process variable (PV) is the value of the driving parameter and it is updated after each flow code iteration. The output, $O$, using the discrete controller equation,

$$
O_{\mathrm{j}}=\mathrm{k}_{\mathrm{p}} \mathrm{e}_{\mathrm{j}}+\mathrm{k}_{\mathrm{i}} \sum_{n=0}^{j} \mathrm{e}_{\mathrm{n}}+\mathrm{k}_{\mathrm{d}}\left(\mathrm{e}_{\mathrm{j}}-\mathrm{e}_{\mathrm{i}-1}\right), \quad \mathrm{e}_{\mathrm{j}}=\mathrm{SP}-\mathrm{PV}_{\mathrm{j}},
$$

adjusts the value of the back pressure boundary condition. Fun3D samples the PV, calculates the error, integral and derivative terms at each iteration j. The boundary condition is updated using Eqn. 14 at a specified (user-defined) interval.

$$
\mathrm{p}_{\text {back,new }}=\left(1-O_{\mathrm{i}}\right) \text { p back,previous }
$$

In this paper, the controller coefficients $k_{p}, k_{i}$, and $k_{d}$ were kept constant for the duration of each simulation. For example, Table 3 lists typical coefficient values used to drive the back pressure using the mass flow rate and AIP Mach number as process variables. The frequency of the update is dependent on the lag time of the simulation in response to

Table 3 Typical controller parameters.

\begin{tabular}{llcccc}
\hline Parameter & $\begin{array}{l}\text { Set } \\
\text { point }\end{array}$ & $\mathrm{k}_{\mathrm{p}}$ & $\mathrm{k}_{\mathrm{i}}$ & $\mathrm{k}_{\mathrm{d}}$ & $\begin{array}{c}\text { Update frequency } \\
\text { (iterations) }\end{array}$ \\
\hline Mass flow & $2.509 \mathrm{~kg} / \mathrm{s}$ & $6.0 \times 10^{-3}$ & $4.0 \times 10^{-4}$ & $1.0 \times 10^{-6}$ & 100 \\
Mach number & 0.6294 & $4.0 \times 10^{-2}$ & $5.0 \times 10^{-2}$ & $1.0 \times 10^{-6}$ & 100 \\
\hline
\end{tabular}

changes in the boundary condition values. Additionally, the period of time required for solution start-up transients to die down can vary problem to problem. Starting the controller too early in the process can unduly influence the behavior of the controller. For this study, 5000 first-order iterations were conducted prior to starting the controller, while the controller frequency update was 2,000 iterations.

\section{Grid Generation}

The participants were provided with a series of grids, both structured and unstructured, to be used for a grid sensitivity study. Additionally, the participants were given the option of generating custom grids under a provided set of guidelines. For the present work, a new series of grids was generated to allow for control of the types of cells used to resolve the boundary layer. The provided unstructured grids were mixed elements due to the use of prisms to resolve the boundary layer. However, the version of USM3D used for the present work required all tetrahedral grids. Note that a set of all tetrahedral grids were provided but the geometry did not include the rakes.

The grid generation was performed using Pointwise ${ }^{\circledR}$ v18.2 [19]. Pointwise ${ }^{\circledR}$ is a structured and unstructured grid generation code with an intuitive interface and CAD clean-up capabilities. The geometry surface is divided into user-defined computational domains. For each domain, the user can define the point density and spacings on the connectors, which define the outer edges of the computational domains. Additionally, Pointwise ${ }^{\circledR}$ offers the user a variety of metrics for controlling the density of the interior of the domains. For unstructured grids, Pointwise ${ }^{\circledR}$ is capable of generating both mixed-element and all tetrahedral grids. The boundary layer is resolved using anisotropic tetrahedrals known as T-Rex cells, which can be combined into prisms to generate mixed-element grids. Grid generation is a two-step process consisting of generating the surface grids and volume grids separately. This allows for more control compared to single step grid generation software. Pointwise ${ }^{\circledR}$ is heavily documented including a variety of tutorials, theory description, and a wide array of scripts available that enable automation of common grid generation problems. 
For the present work, Pointwise ${ }^{\circledR}$ was used to generate a mixed-element coarse grid for the s-duct geometry. The coarse grid was developed with the goal of matching the grid resolution to that of the provided coarse grids. Once the coarse grid was generated, the medium and fine grids were created using the gridRefine script available on GitHub. The gridRefine script was developed by Pointwise ${ }^{\circledR}$ and provides an automated way to perform grid refinement based on a user-defined scale factor. The scale factor is uniformly applied to both the connectors and the interior of the domains. For the connectors, the scale factor is applied to refine the spacing constraints and point densities. In the domain interior, the scale factor is applied to the edge lengths of the cells. Additionally, the user has the option of applying the scale factor to the $y+$ value to refine the boundary layer. For the present case, $y+$ was held constant at a desired value of 1 to be consistent with the provided grids. A scale factor of $2^{1 / 4}$ was found to provide comparable densities to that of the provided grids for this work.

Once the new series of mixed-element grids was generated, an all-tetrahedral series was generated for the USM3D solver. Pointwise ${ }^{\circledR}$ allows the user to choose the type of elements used in the boundary layer when solving the grid volumes. For the mixed-element grids, the boundary layer consists of anisotropic tetrahedrals that are then recombined into prisms. For the all-tetrahedral grids, this recombination step is omitted. The result is that the grids only differ in the types of cells in the boundary layer. Illustrations of the mixed-element and tetrahedral-element grids are provided in Figures 3-6. Additionally, the grid resolution of the coarse, medium, and fine grids are provided for both the mixed-element and all-tetrahedral grids in Table 4.

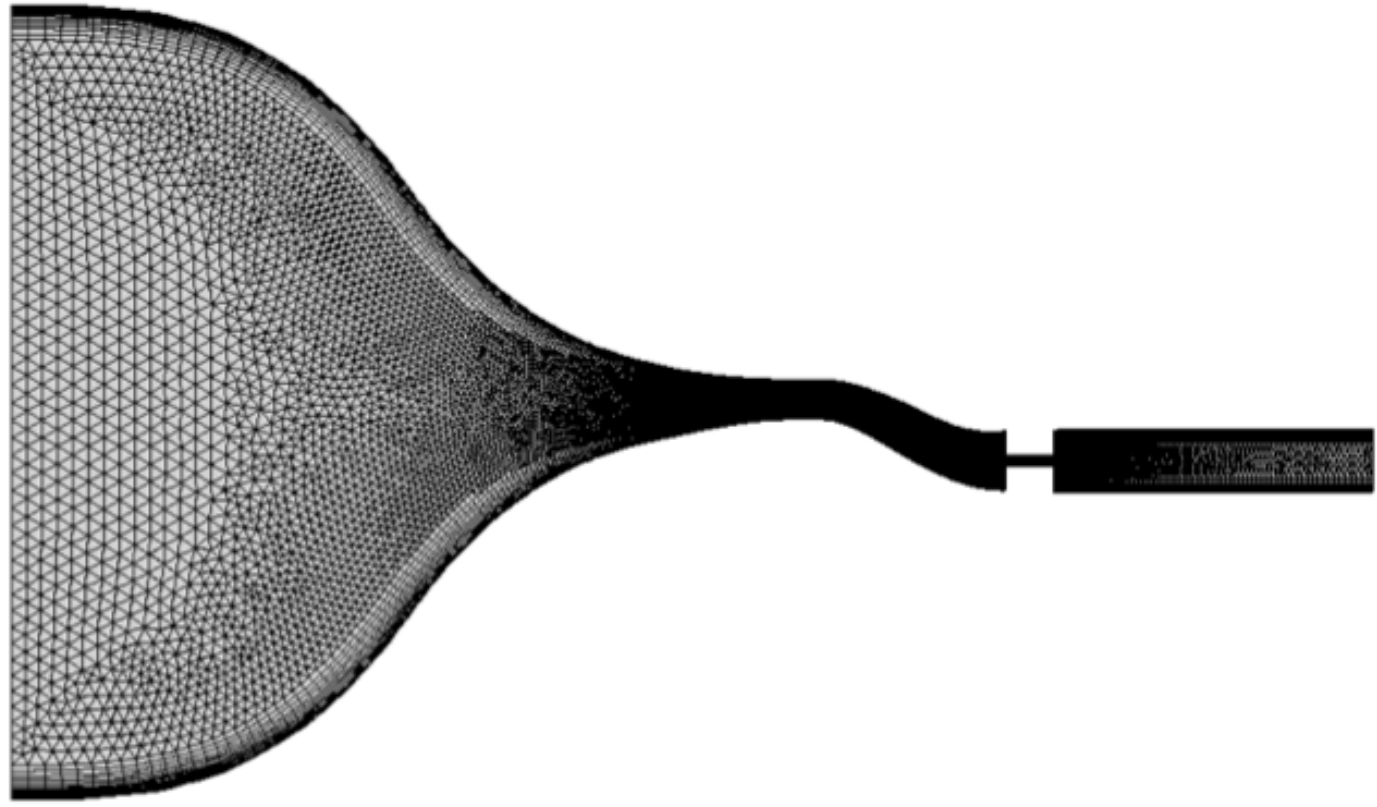

Fig. 3 Symmetry plane for coarse, mixed-element grid.

Table 4 Grid resolutions.

\begin{tabular}{lccc}
\hline & \multicolumn{3}{c}{ Nodes / Cells (Millions) } \\
\hline & Coarse & Medium & Fine \\
\hline Mixed-Element Grids & $4.799 / 11.999$ & $6.706 / 17.979$ & $9.268 / 26.087$ \\
Tetrahedral-Element Grids & $4.799 / 27.843$ & $6.706 / 38.176$ & $9.268 / 52.162$ \\
\hline
\end{tabular}

Note that since the all-tetrahedral grids do not undergo the recombination step, there are significantly more cells in the boundary layer compared to the mixed-element grids. The number of nodes remain relatively unchanged such that the grid resolution is the same if both grids were used in a node-based solver. However, USM3D not only requires 


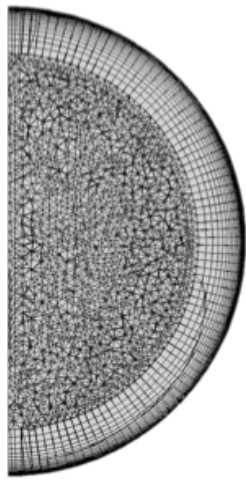

(a) Coarse.

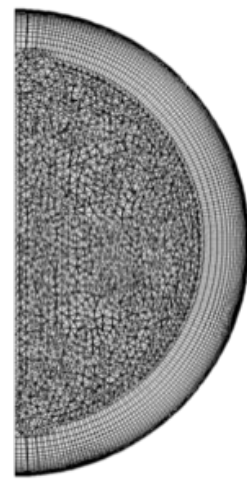

(b) Medium.

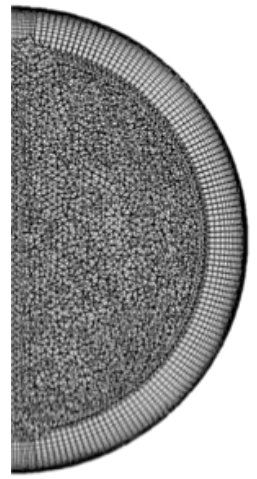

(c) Fine.

Fig. 4 Resolution at AIP for mixed-element grids.

all-tetrahedral grids, but it is also a cell-centered solver. The result is that the USM3D grids are significantly more refined than the Fun3D grids. Because of this, the FUn3D and USM3D results cannot be directly compared and are instead treated as separate analyses. A more valuable comparison between the two solvers could be obtained if the grid resolution were more closely matched. In previous work, this has been performed by attempting to match the number of cells in the USM3D grids to the number of nodes in the Fun3D grids. However, a single set of grids was provided to the participants regardless of whether the solver being used was node-based or cell-centered. In an effort to be consistent with the workshop, the grids were developed in the manner discussed here. This issue will be partly resolved for future workshops with the release of the mixed-element version of USM3D [20].

\section{Studies}

As part of this work, some preliminary studies were performed to identify the best solution strategy for the s-duct analyses. Note that all of the present analyses were performed using Fun3D. Additionally, the outflow boundary condition was set to have a fixed back pressure. The mass flow rate was not specifically targeted to eliminate any effects of a varying outflow condition. The main objectives of the preliminary studies were to assess the applicability of: 1) a steady solution strategy and 2) a half span geometry. The details of the preliminary studies are provided in the following discussion.

\section{Steady vs. Time-Accurate}

Based on previous PAW analyses, the flow at the AIP contains a separated region, which is inherently an unsteady phenoma. The objective of the first study was to determine if a steady solution strategy would be sufficient for this problem containing separated flow. The study consisted of performing both steady and time-accurate simulations. The time step used for the time-accurate simulations was chosen to be 0.00015 seconds, which corresponds to $1 / 100$ th of the time required for a particle moving at a Mach number of 0.6294 to travel the length of the s-duct geometry. For each time step, 50 subiterations were performed to ensure adequate convergence. Additionally, the simulations were performed using the full span geometry. The steady and time-averaged pressure recovery contours are provided in Fig. 7.

The results show that there is a significant difference in the flow structure at the AIP for the steady and time-averaged solutions. Additionally, the results show that while there is little difference between the $P R$ values, the $D P C P_{\text {avg }}$ values are significantly different. It appears that this flow is unsteady and that a steady simulation strategy is not sufficient. However, one significant finding during these preliminary analyses was that the application of a flux limiter damps out some of the unsteadiness allowing for a steady simulation to provide comparable flow structures to the time-averaged result. The results in Fig. 8 show a comparison between the $P R$ contours at the AIP for the case of a steady simulation using the minmod flux limiter and a time-averaged simulation with no flux limiter. The flow structures look remarkably similar between the two solutions. However, the $P R$ and $D P C P_{a v g}$ values are different. For the present case, the maximum Mach number was on the order of 1.0 such that it is possible that the flux limiter was changing the flow solution near shocks forming in the rake, which was causing differences upstream. To test this, another case was 


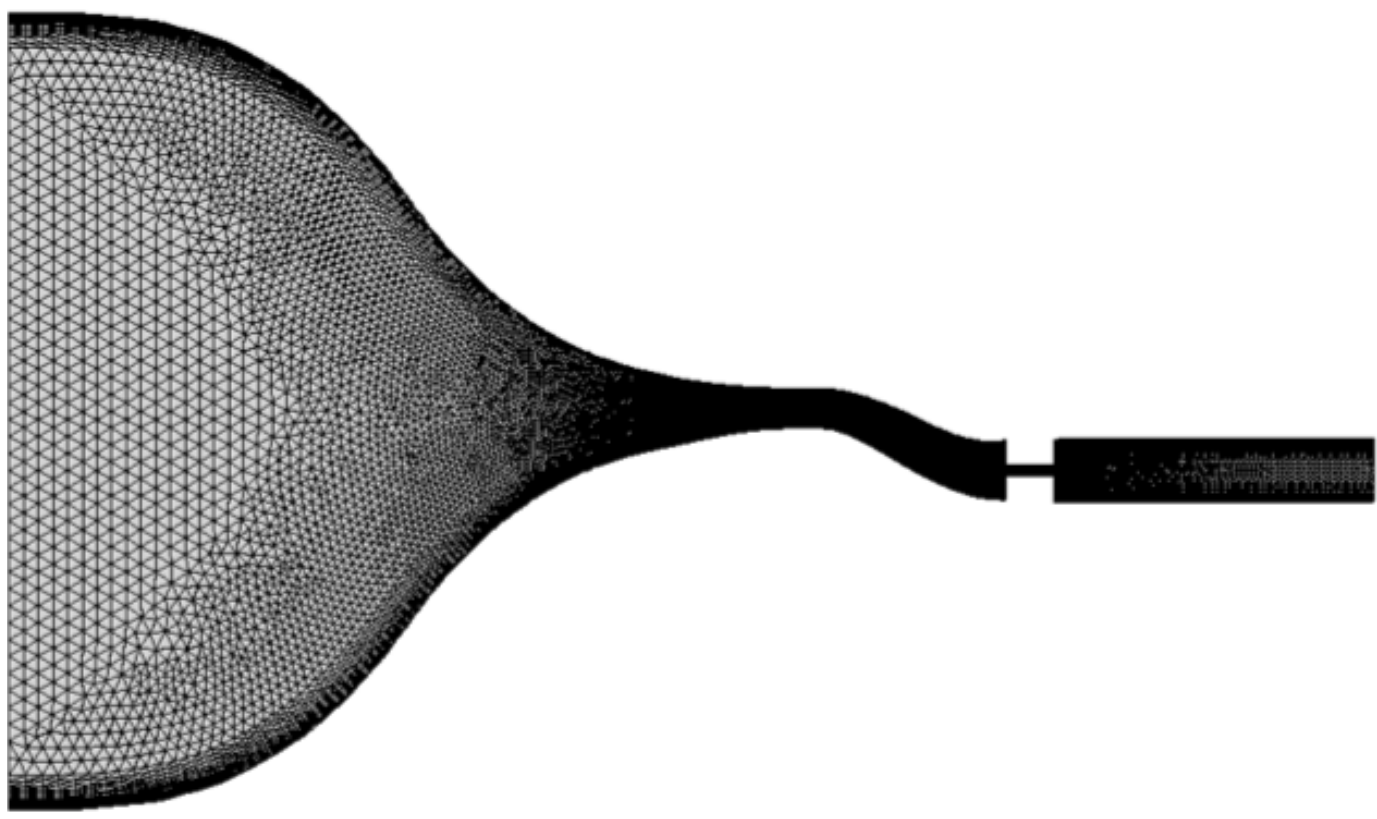

Fig. 5 Symmetry plane for coarse, tetrahedral-element grid.

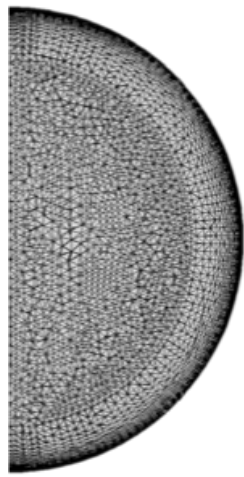

(a) Coarse.

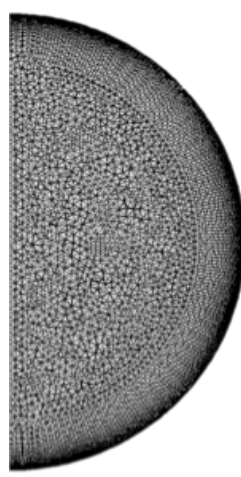

(b) Medium.

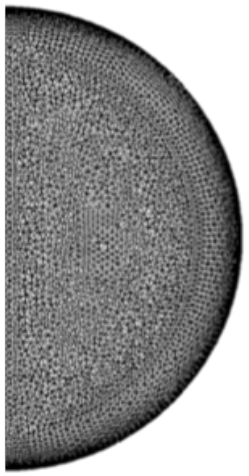

(c) Fine.

Fig. 6 Resolution at AIP for tetrahedral-element grids.

performed with a significantly lower Mach number to see if the flux limiter was still causing a difference in the solutions. The results of this analysis are provided in Fig. 9. 


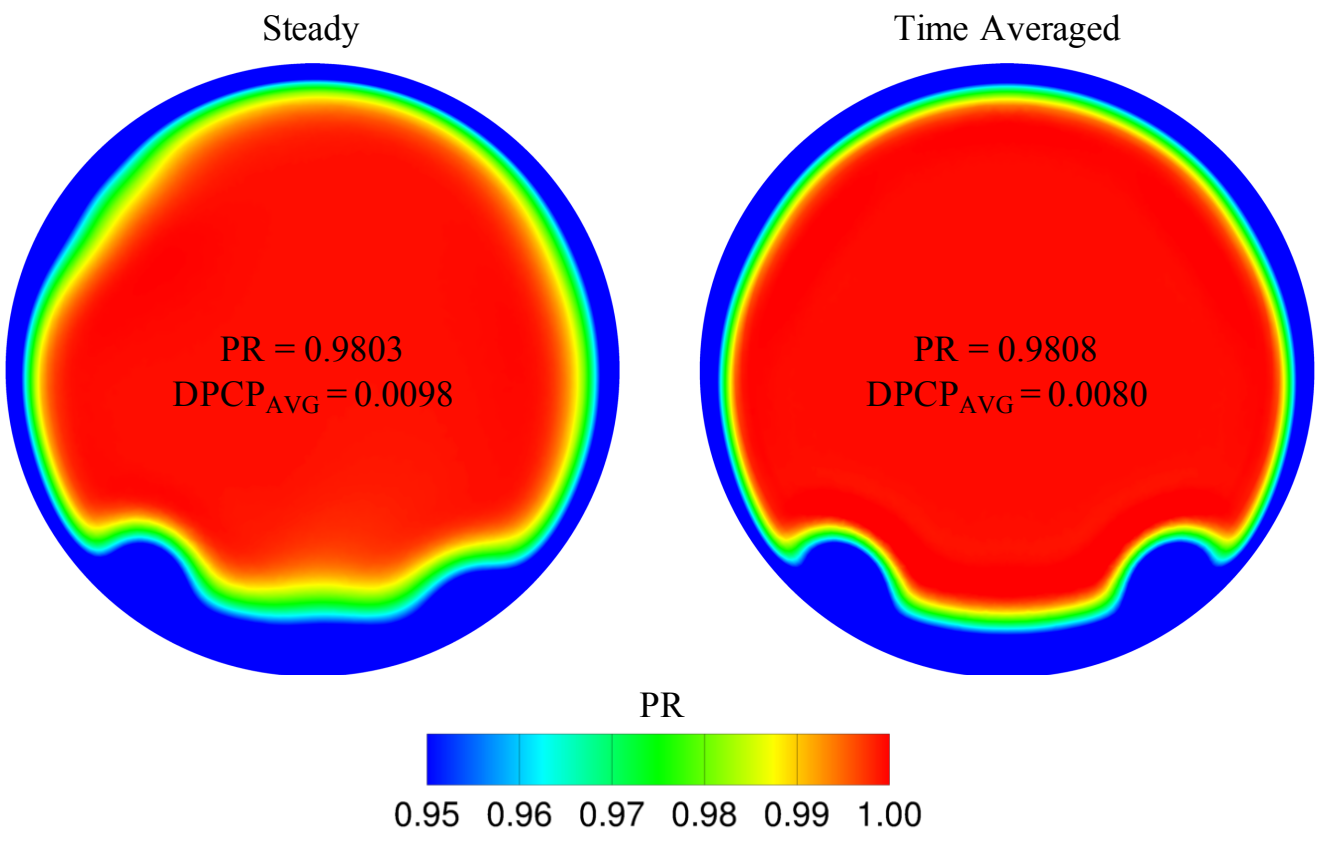

Fig. 7 Steady vs. Time Averaged pressure recovery at AIP obtained from Fun3D.

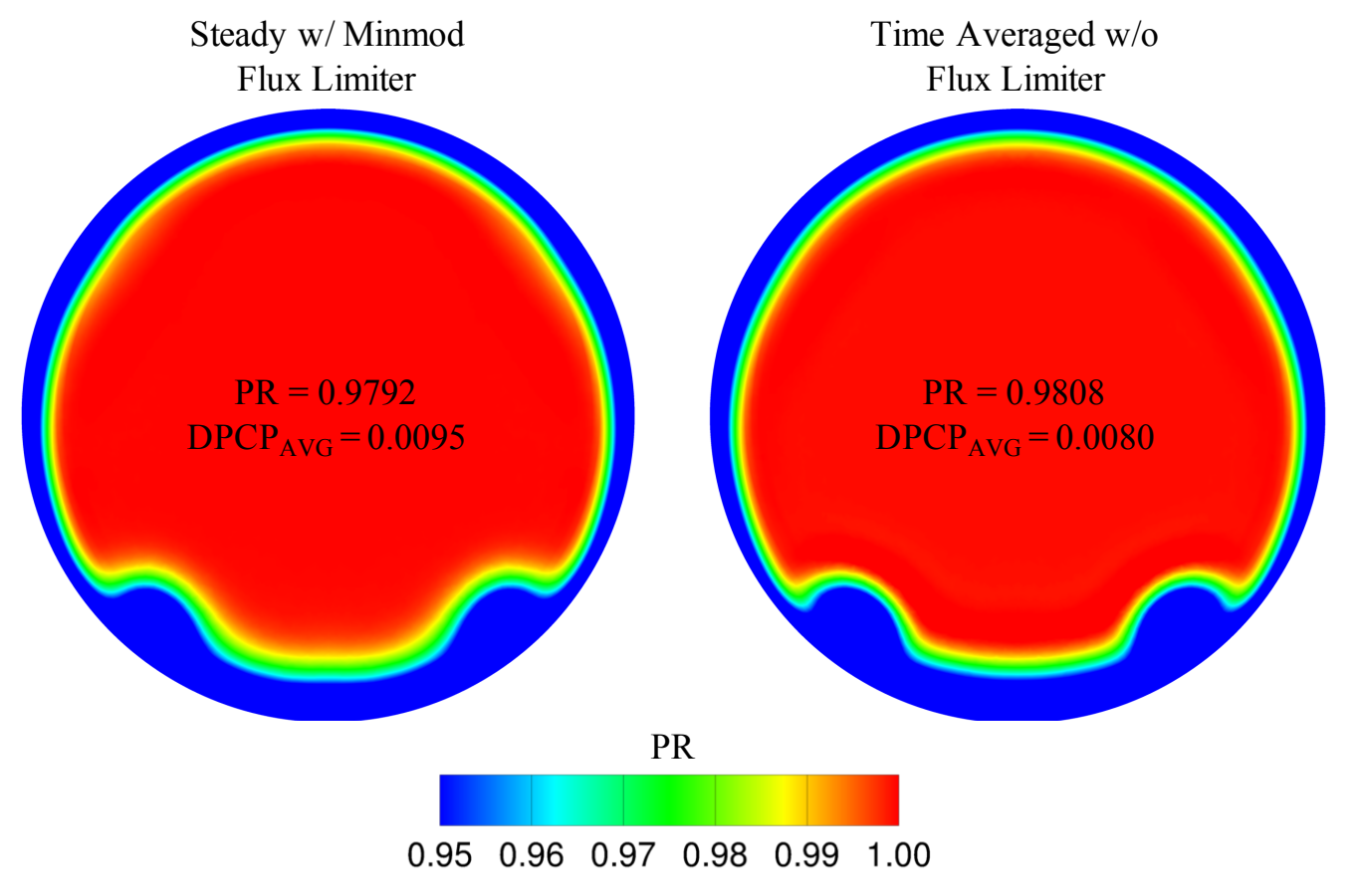

Fig. 8 Steady w/ limiter vs. Time Averaged w/o limiter pressure recovery at AIP obtained from Fun3D. 


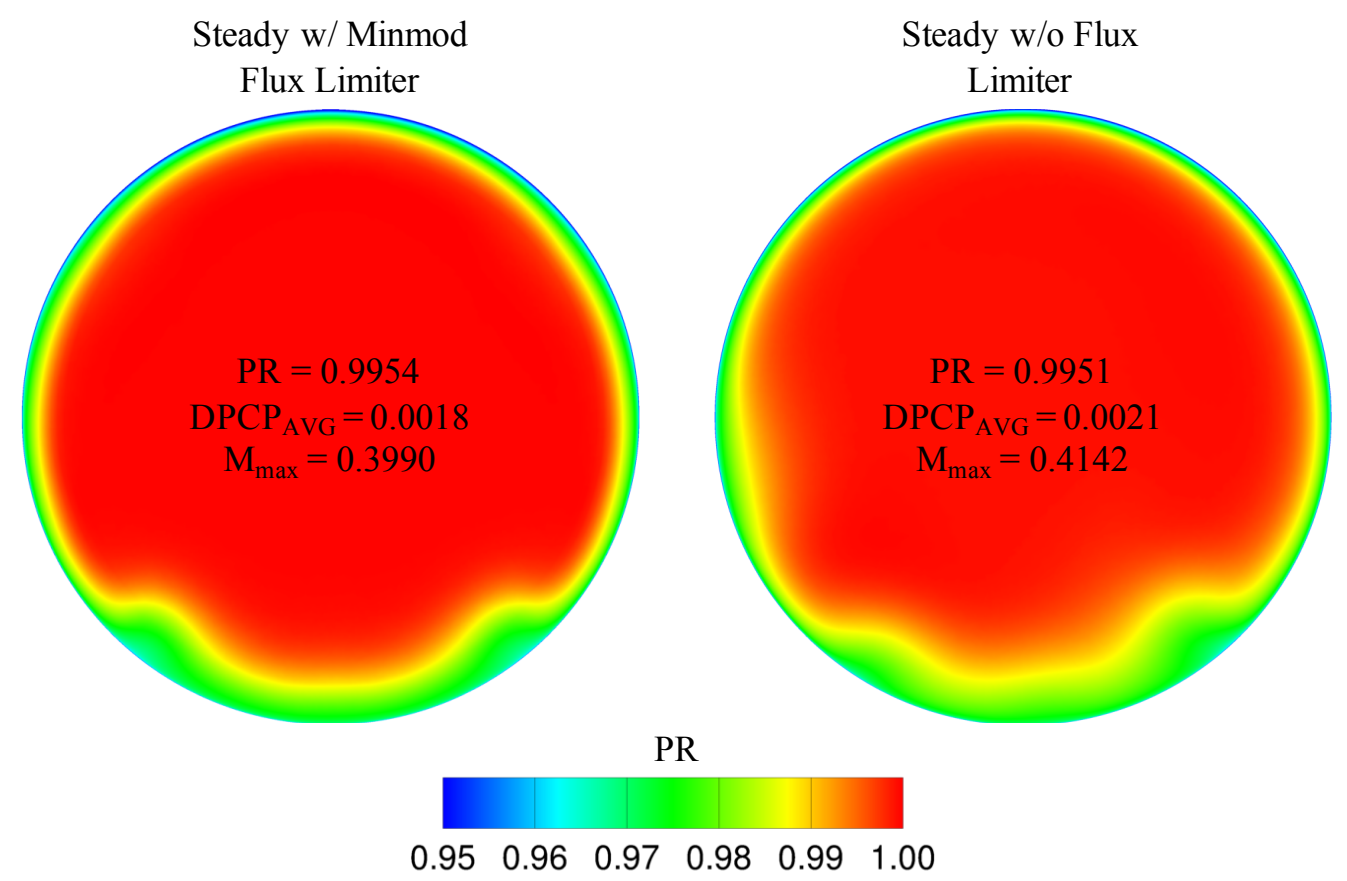

Fig. 9 Steady w/ limiter vs. w/o limiter pressure recovery at AIP obtained from Fun3D.

The results show that even with a flow containing a maximum Mach number of roughly 0.4, the flux limiter appears to be changing the flow solution at the AIP. In theory, the flux limiter should only become active near shocks. However, the present analysis shows that it can also become active and operate on gradients in flows not containing shocks. This result requires further exploration. Based on the results of this analysis, the appropriate solution strategy was determined to be a time-accurate simulation without the use of a flux limiter. Please note, the USM3D full-span steady solutions did not show the same asymmetry as seen with Fun3D when the back pressure was manually adjusted. Consequently, all of the USM3D solutions are steady state. An additional study would be to better integrate the USM3D controller then run both steady and unsteady cases to see if the FUN3D results are duplicated.

\section{Half-vs. Full-Span Simulation}

The workshop grids for the s-duct geometry were provided as half span. The second study was performed to assess the applicability of a half-span simulation for the s-duct geometry. The results are provided in Fig. 10.

Based on the results of this study, the half-span simulation provides the same predicted values of $P R$ and $D P C P_{a v g}$ and therefore, is applicable to this analysis. The half-span geometry was used for all of the remaining results discussed in this paper. Note that this study was originally motivated by differences observed in the half and full span steady flow simulations. The steady flow simulation for the full span geometry provided in Fig. 7 showed that the flow separation was asymmetric and that a half-span geometry would not be sufficient. However, the time-accurate results mitigated this issue and allowed for the use of a half span geometry for the analyses discussed in this paper.

\section{Results}

\section{A. CFD2EXP Results}

\section{Convergence}

The goal of the CFD2EXP part of the workshop was to match the experimental data taken at the AIP. Both the internal Fun3D and external USM3D controllers probe the 40 points that made up the AIP and computed Mach number to compare to the desired value of 0.6294. Both controllers then changed the back pressure setting at the downstream boundary to attempt to match the Mach number. Figures 11 and 12 show the results for Fun3D and USM3D, respectively. 

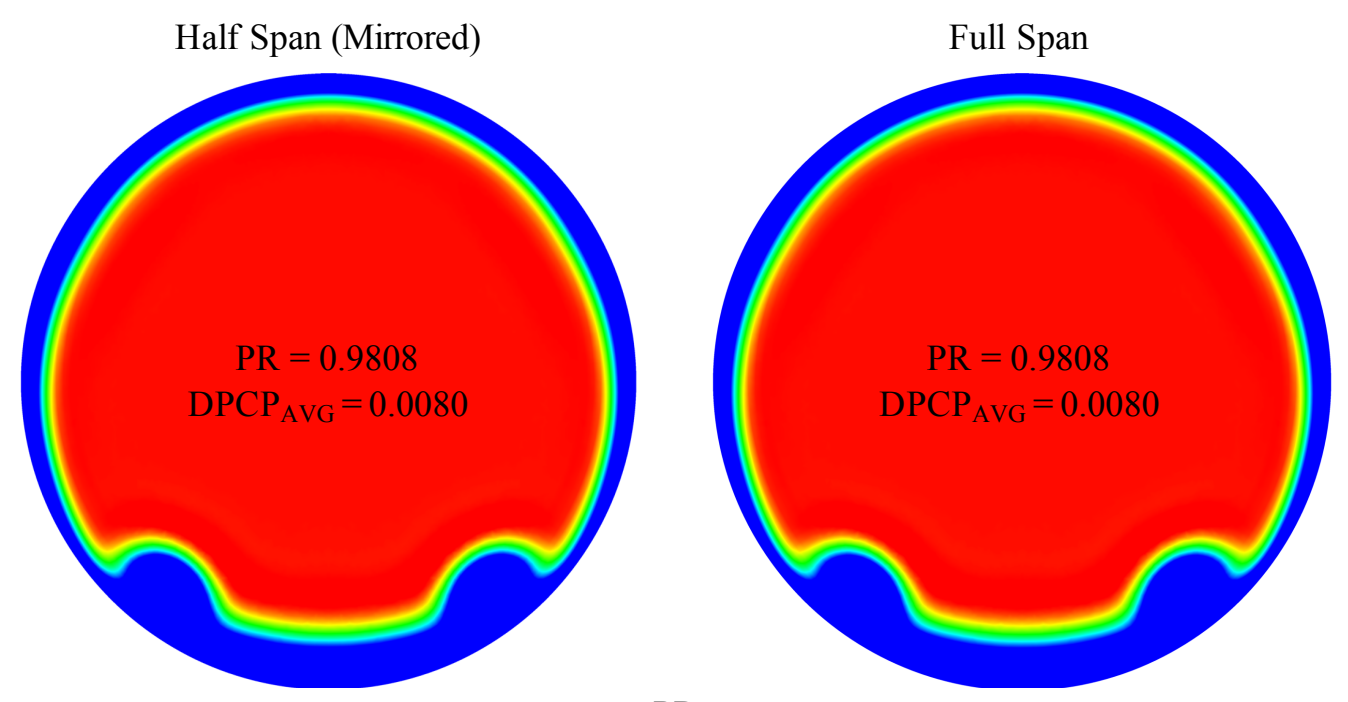

PR

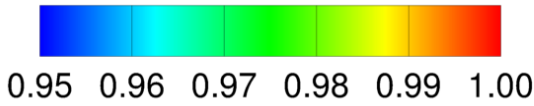

Fig. 10 Full span vs. half span pressure recovery at AIP obtained from Fun3D.

Fun3D, which was run time-accurate, had 200 subiterations in each iteration with the controller changing the back pressure every ten outer iterations for a total of 2,000 iterations between updates. The USM3D solution also had 2,000 CFD iterations between each controller iteration. Also, the controller was not started until a steady solution was obtained (approximately 30,000 iterations for the example shown). Both figures show that the controllers overshot their target but were able to make the appropriate adjustments back. Additionally, small changes were made toward the end in order to better match the desired Mach number.

Figures 13 and 14 provide additional insight into the Fun3D and USM3D results. Fun3D subiterations show that both the mean flow and turbulence drop considerably in the first 0.1 fractional time step then, in the case of the mean flow, slowly decrease as the subiterations continue. Both drop more than considered necessary for subiteration convergence. For USM3D, the overall plot of the mean flow and turbulence residuals are shown since it was run steady state. The first 30,000 iterations show both numbers reducing to normal convergence levels. After this, the controller changed the back pressure and a series of spikes is observed every 2,000 iterations until the changes became minimal. Overall, the USM3D residuals stay within the expected band for a converged solution. 


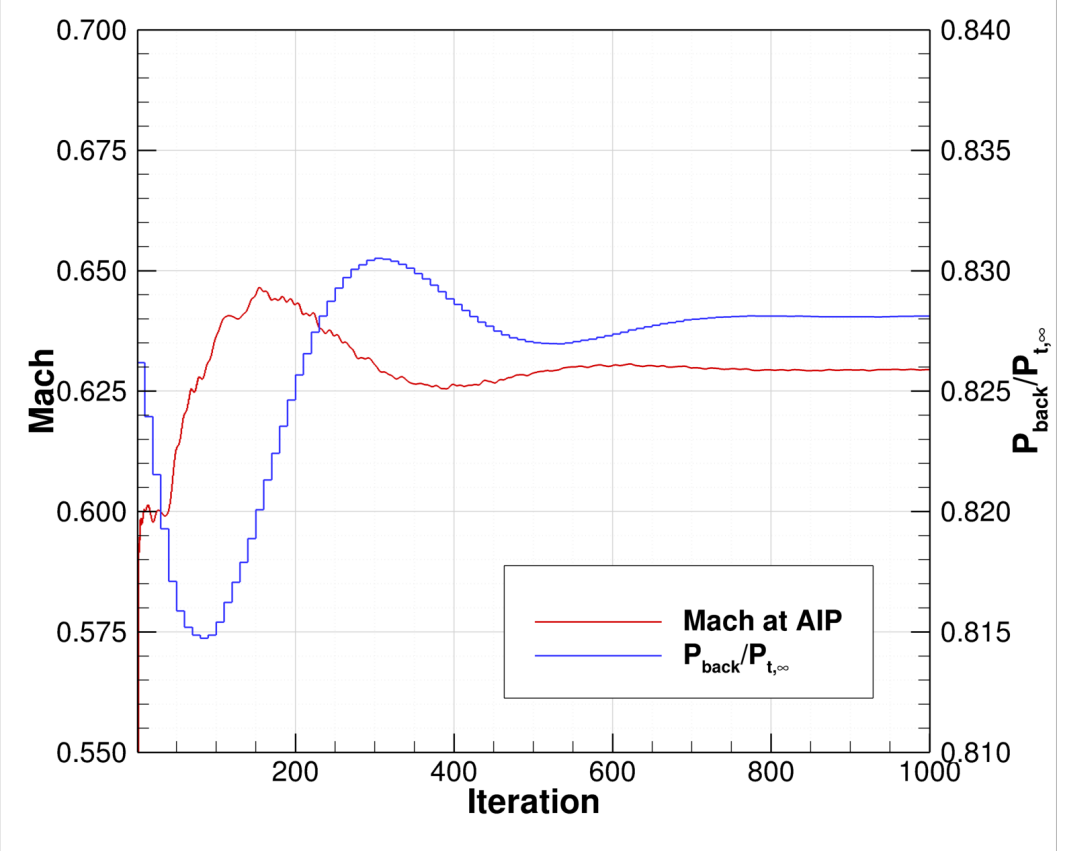

Fig. 11 Controller history for CFD2EXP Fun3D simulations.

\section{Grid Refinement Study}

As previously discussed, a series of grids comparable to the grids provided by the workshop were created for this study. Table 4 summarizes the sizes of the Fun3D (mixed-element) and the USM3D (tetrahedral) grids. Again, since the grids were made to match the workshop and not be consistent resolutions between the two solvers, a Fun3D to USM3D comparison would be inappropriate. The grid refinement study results for the two solvers will be discussed separately.

\section{Fun3D}

Figure 15 compares the Fun3D AIP solutions at the three different grids levels. Qualitatively, the separation bubbles on the bottom of the duct become better defined and grow slightly in size, as the grid is further refined. Quantitatively, $P R$, which is also shown in Fig. 16, increases slightly with each grid refinement. DPCP, Fig. 17, is highly sensitive to grid resolution, especially when the data are taken at the 40-probes only. The shifting of the separation bubbles show this sensitivity although all the changes occur at the thousandths value.

\section{USM3D}

Figure 18 compares the USM3D AIP solutions at the three different grids levels. Qualitatively, the separation bubbles on the bottom of the duct vary significantly more than observed with Fun3D. It is not until the fine grid that the flow separation exhibits the bubbles that were observed for the Fun3D solutions. Additionally, USM3D predicts a considerably thicker boundary layer, which may be due to the grid being used. Quantitatively, $P R$, which is also shown in Fig. 19, is different than the other two grids due to the extended separation region on the sides of the duct. DPCP, Fig. 20, follows the same pattern as the $P R$ plot, by that the medium grid shows the most distortion, although again, all the changes occur at the thousandths value. 


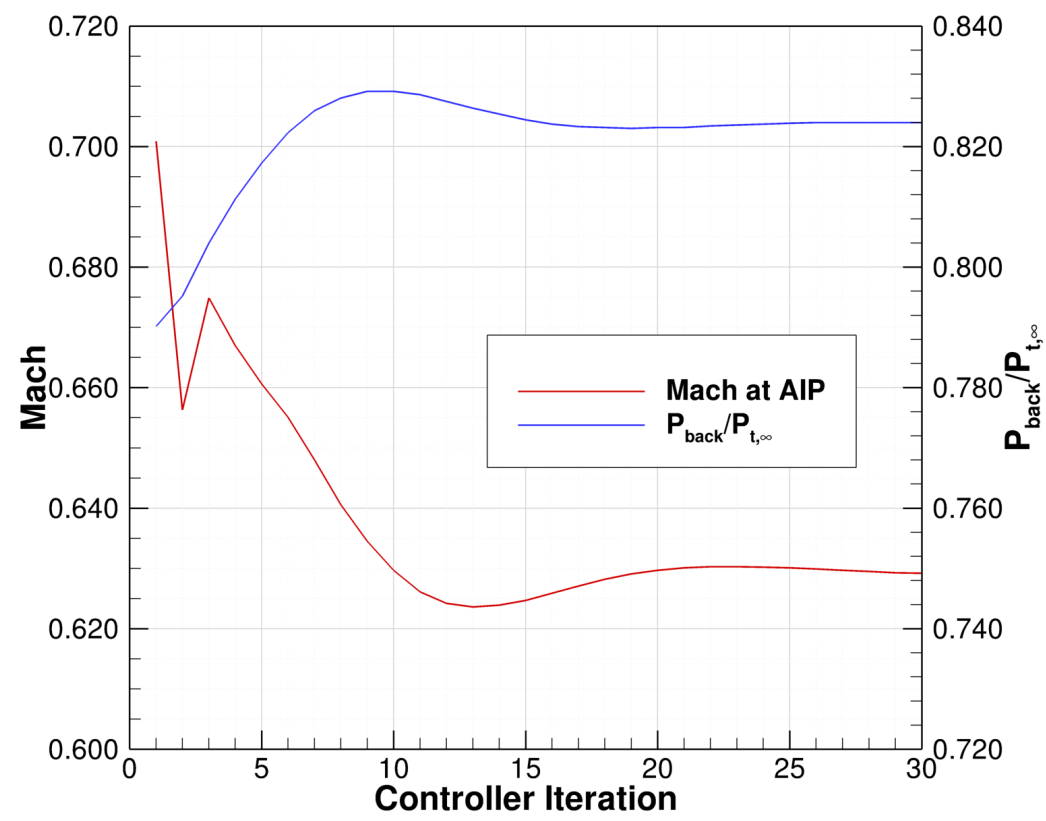

Fig. 12 Controller history for CFD2EXP USM3D simulations.

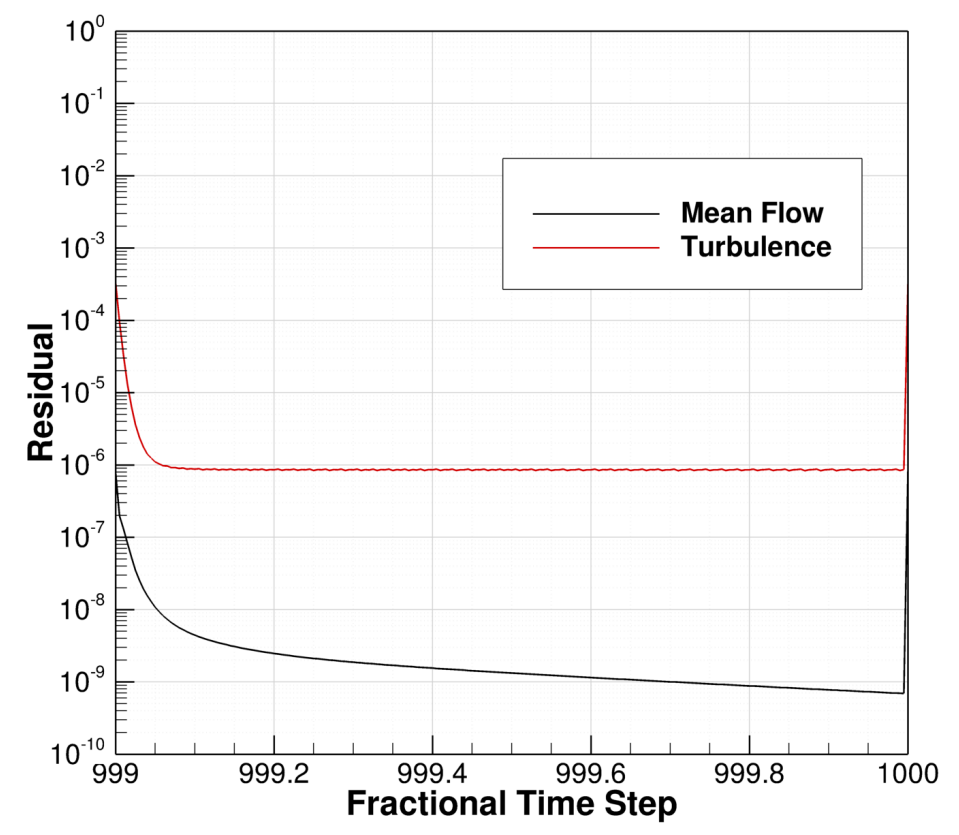

Fig. 13 Typical subiterative convergence for CFD2EXP simulations. 


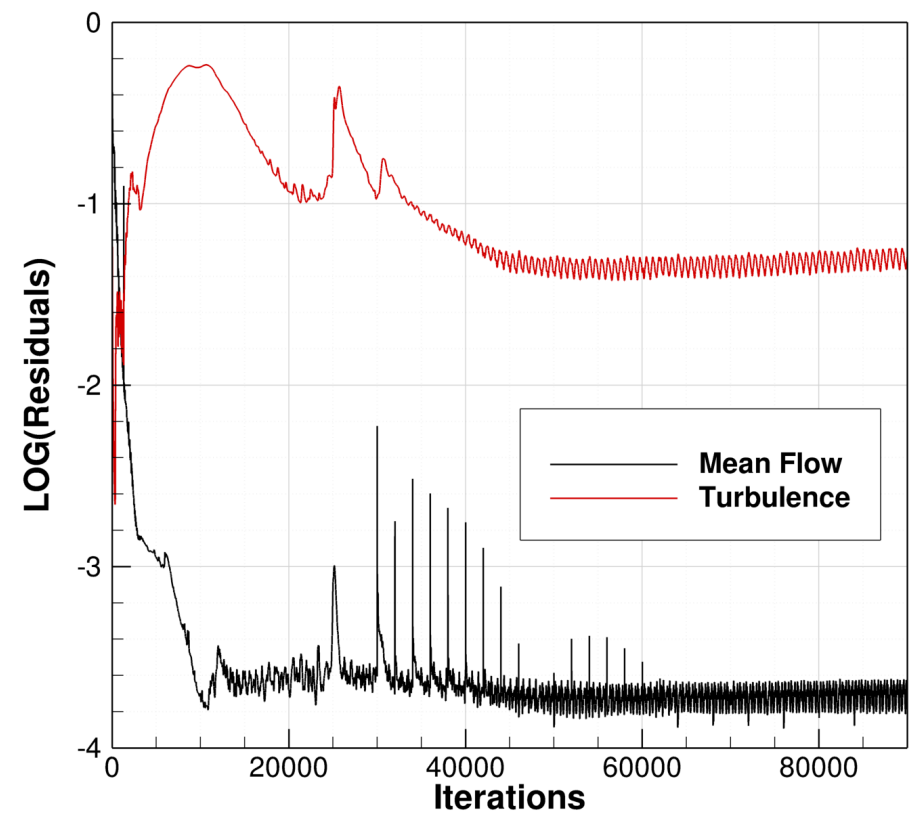

Fig. 14 Typical convergence for USM3D simulations.
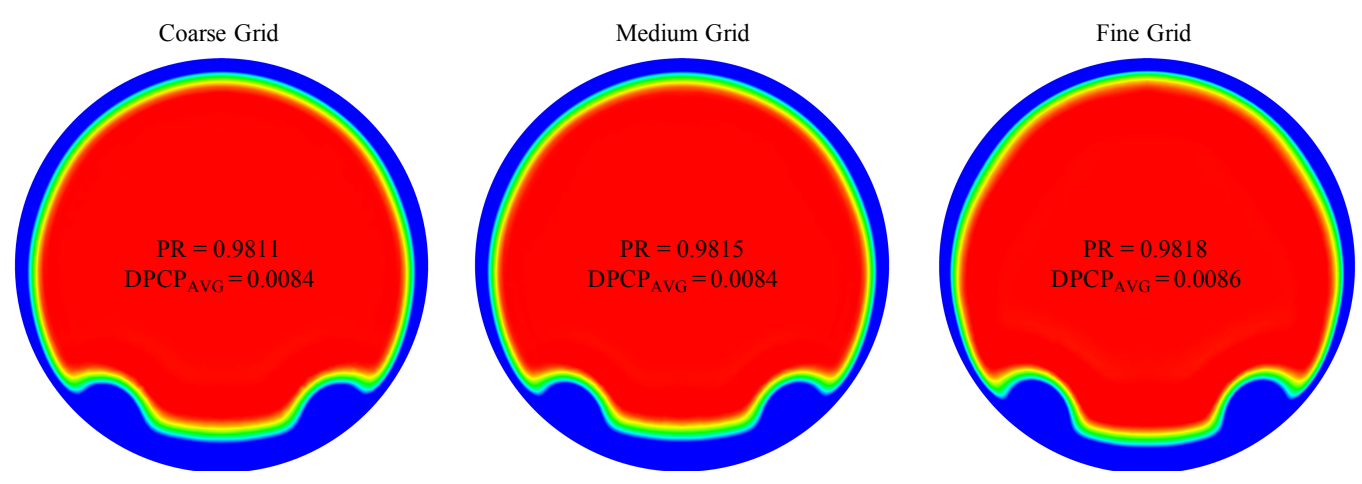

PR

$\begin{array}{llllll}0.95 & 0.96 & 0.97 & 0.98 & 0.99 & 1.00\end{array}$

Fig. 15 Pressure recovery at AIP obtained from Fun3D for coarse, medium, and fine grids. 


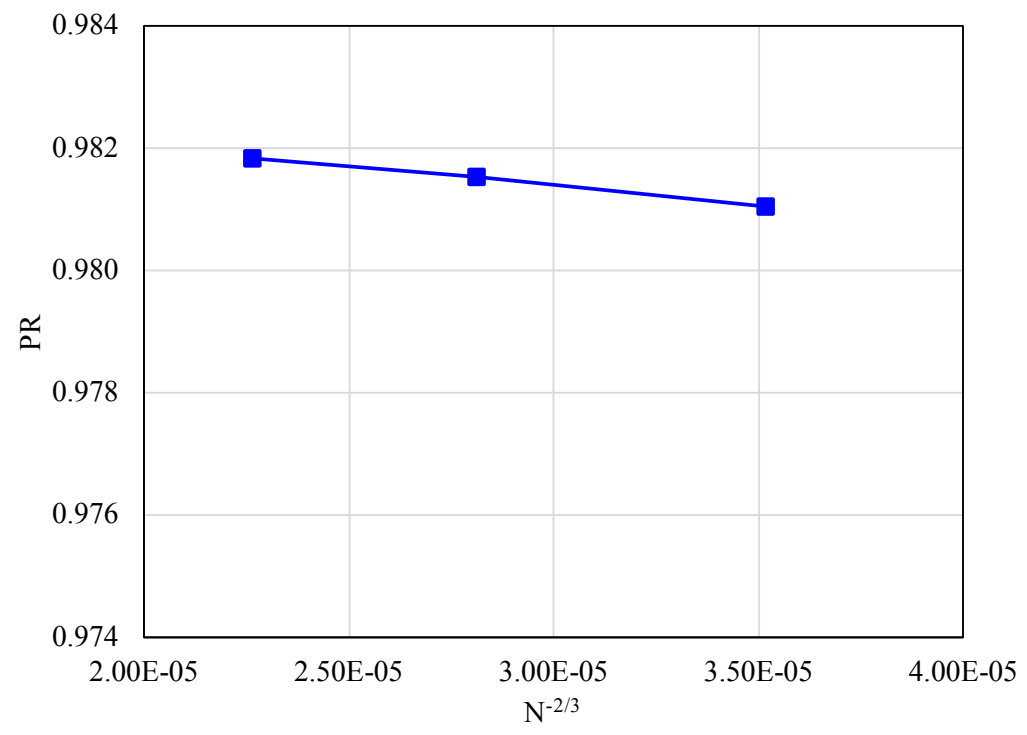

Fig. 16 Pressure recovery at AIP obtained from Fun3D as a function of grid refinement.

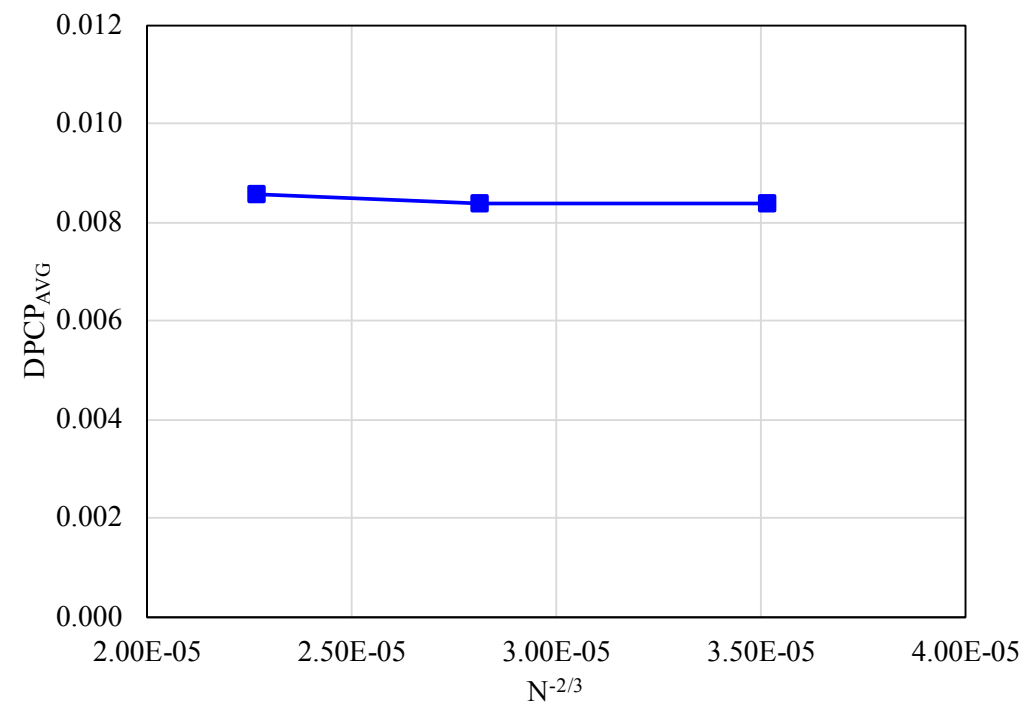

Fig. 17 DPCP at AIP obtained from Fun3D as a function of grid refinement. 


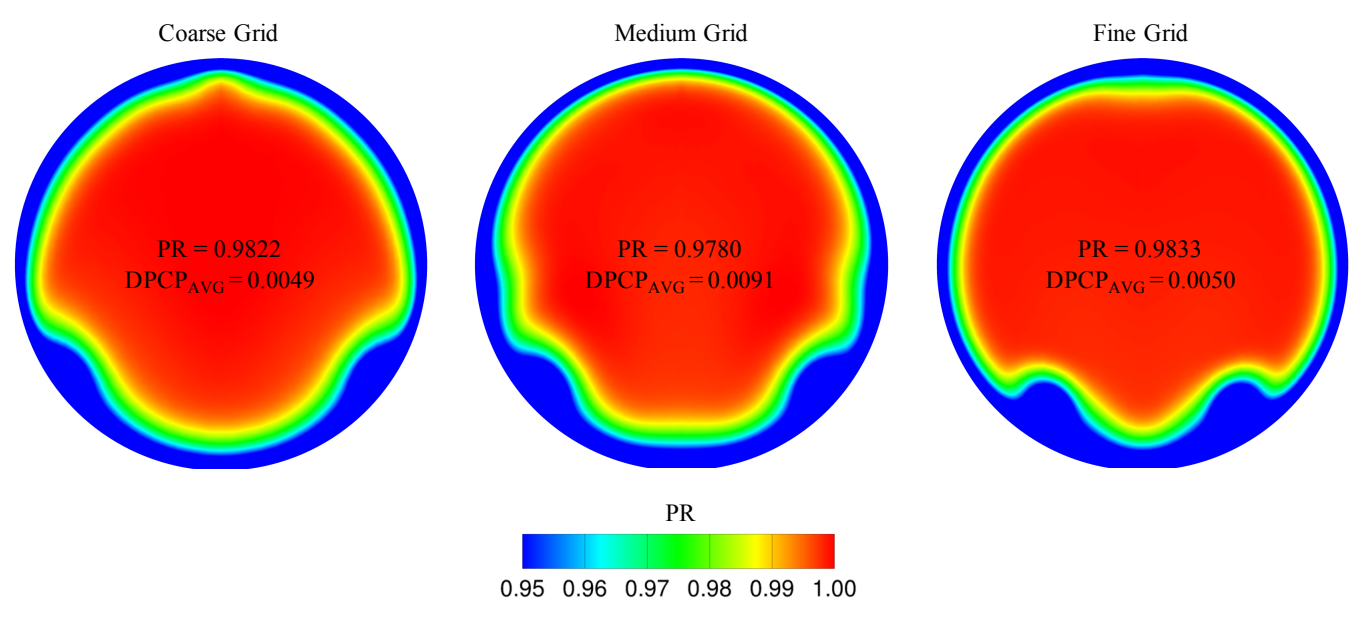

Fig. 18 Pressure recovery at AIP obtained from USM3D for coarse, medium, and fine grids.

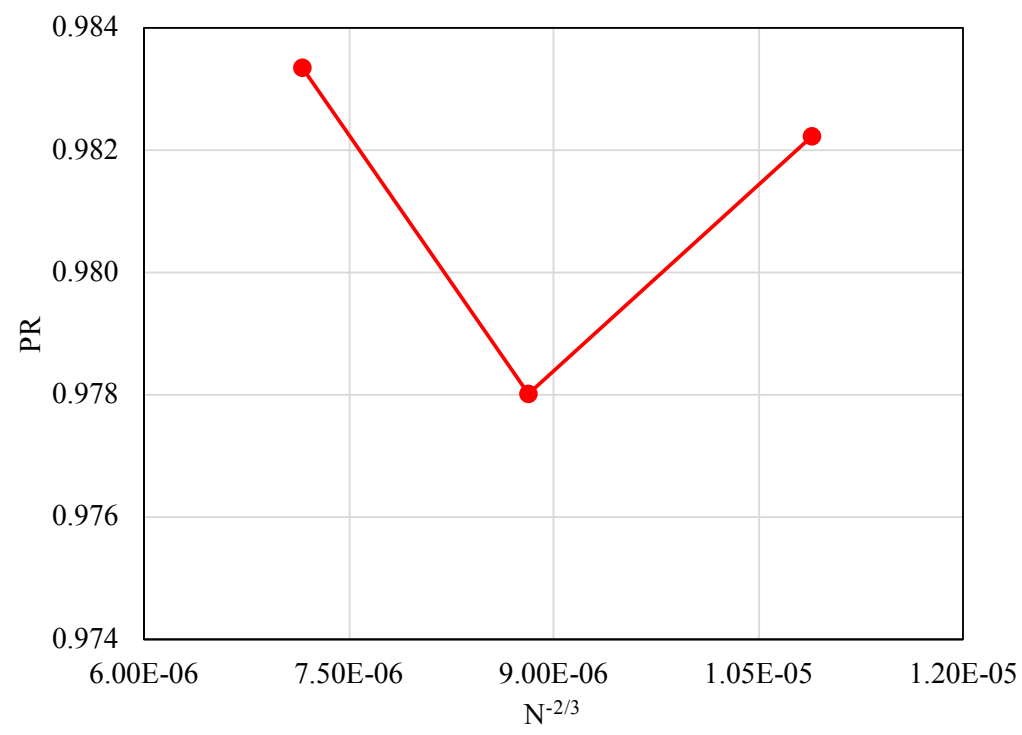

Fig. 19 Pressure recovery at AIP obtained from USM3D as a function of grid refinement. 


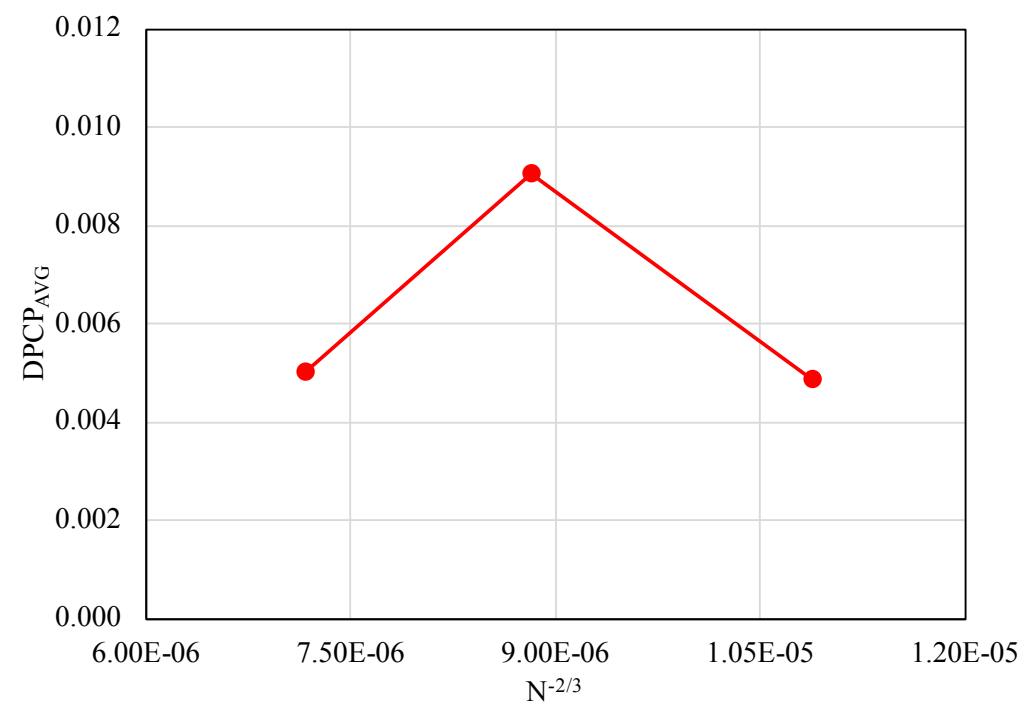

Fig. 20 DPCP at AIP obtained from USM3D as a function of grid refinement.

\section{Turbulence Model Study}

Figure 21 compares the Fun3D AIP solutions for two different turbulence models. The separation bubbles on the bottom of the duct have diffused more with use of the k- $\omega$-SST turbulence compared to the Spalart-Allmaras model with the QCR correction (SA-QCR). The persistence of cross-flow vortices would be stronger with the nonlinear Reynolds stress contributions in the SA-QCR model. Despite the nuance in the shapes of the flow distortion in the lower portion of the duct, the pressure recovery and distortion numbers are fairly close, with slightly over $0.5 \%$ variation in PR and $1 \%$ variation in distortion intensity.

\section{B. CFD2CFD Results}

Only Fun3D results will be presented in this paper for the CFD2CFD study.

\section{Convergence}

As previously discussed, the CFD2CFD analyses were performed by prescribing the mass flow rate at the outflow boundary as opposed to targeting the mass flow rate at the AIP directly. The differences in methods used to calculate the mass flow rate result in larger mass flow rates for the CFD2CFD analyses relative to the CFD2EXP analyses. For the CFD2EXP cases, the averaged 40 probe data are used along with Eqs. 9-11 to calculate the mass flow rate at the AIP while the integration provided in Eqn. 15 is performed over the full grid resolution to obtain the mass flow rate for the CFD2CFD cases. An example of the calculated mass flow rates using the two described methods for the Fun3D coarse grid are provided in Table 5.

$$
\dot{\mathrm{m}}=\int_{\Omega} \rho V d A
$$

Table 5 Mass flow rate differences for integrated and calculated methods.

\begin{tabular}{lc}
\hline & Mass flow rate $(\mathrm{kg} / \mathrm{s})$ \\
\hline Integrated & 2.501 \\
Calculated & 2.649 \\
\hline
\end{tabular}



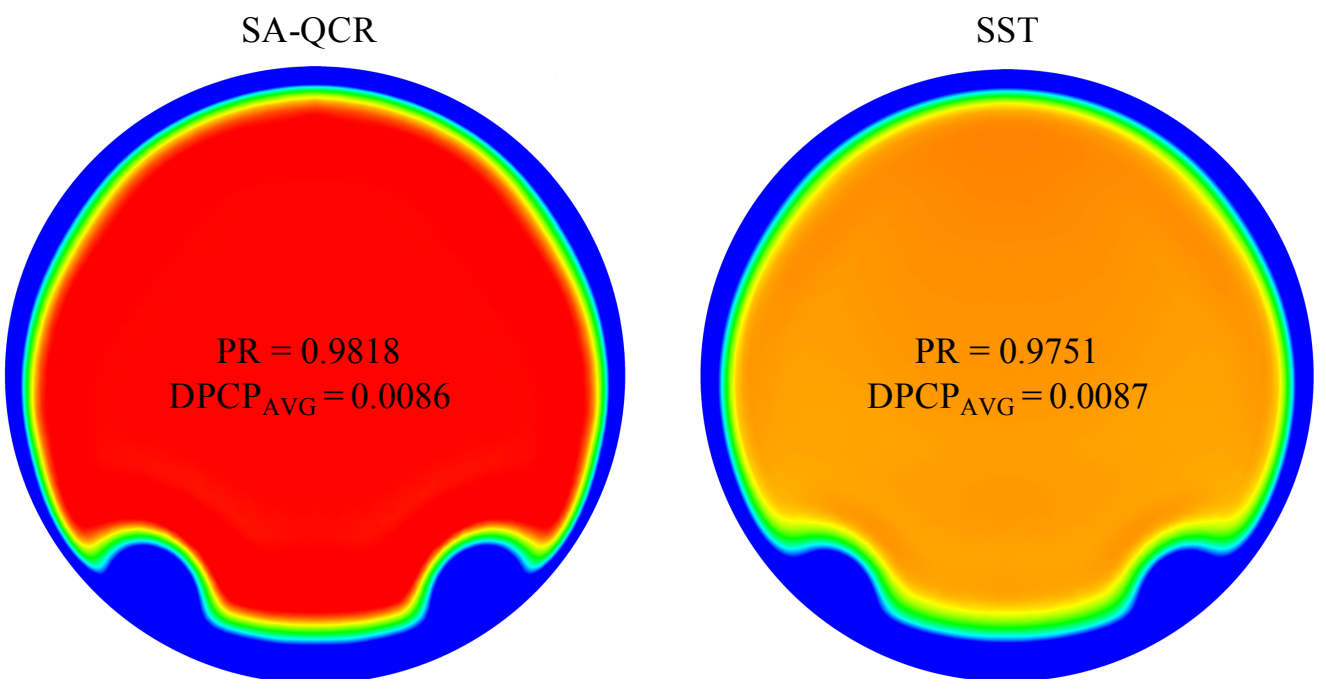

PR

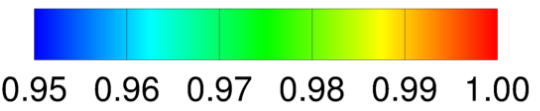

Fig. 21 Comparison of pressure recovery at AIP obtained from Fun3D using SA-QCR and SST turbulence models.

The observed increase in mass flow rate for the CFD2CFD analyses also results in a higher Mach number at the AIP resulting in difficulties due to the presence of supersonic flow as the flow passes through the rake. The mass flow boundary condition in Fun3D used for these calculations consists of a simplified P-controller that adjusts the back pressure at the outflow using a relaxation approach when attempting to attain the requested mass flow rate. The PID-controller discussed earlier in this paper, has this functionality as well, but was not used for the CFD2CFD study. For the present problem, the flow solution starts out normally. The mass flow rate is lower than the specified value so the controller attempts to increase the mass flow rate by decreasing the back pressure at the outflow boundary. Eventually, the flow begins to behave like a choked flow. The back pressure continues to be reduced because the mass flow rate is too low but the mass flow rate essentially remains unchanged. An illustration of this behavior is presented in Fig. 22. Also, the presence of supersonic flow in the rake is illustrated in Fig. 23.

Because of this behavior, the final solution was chosen to correspond to the timestep just prior to the choked flow behavior. Future workshops will need to look into the CFD2CFD comparisons further to determine an appropriate mass flow rate that does not lead to the behavior shown here. An illustration of the subiterative convergence for this case is provided in Fig. 24. It is also for this reason, that the USM3D external controller would have had difficulties with this case and its data were excluded from this section.

\section{Grid Refinement Study}

Figure 25 compares the Fun3D AIP solutions at the three different grids levels. Qualitatively, the separation bubbles on the bottom of the duct become better defined and grow slightly in size, as the grid is further refined. Quantitatively, $P R$, which is also shown in Fig. 26, increases slightly with each grid refinement. DPCP, Fig. 27, is highly sensitive to grid resolution, especially when the data are taken at the 40-probes only. The shifting of the separation bubbles show this sensitivity although all the changes occur at the thousandths value.

\section{Turbulence Model Study}

Figure 28 compares the Fun3D AIP solutions for two different turbulence models at the experimental mass flow rate applied at the CFD outflow boundary. The flow distortion regions are notably different for this running condition. The separation bubbles on the bottom of the duct using the $\mathrm{k}-\omega-\mathrm{SST}$ model are more diffused by comparison with the SA-QCR simulation, seemingly similar to the previous turbulence model comparison, as shown in Fig. 21. The pressure 


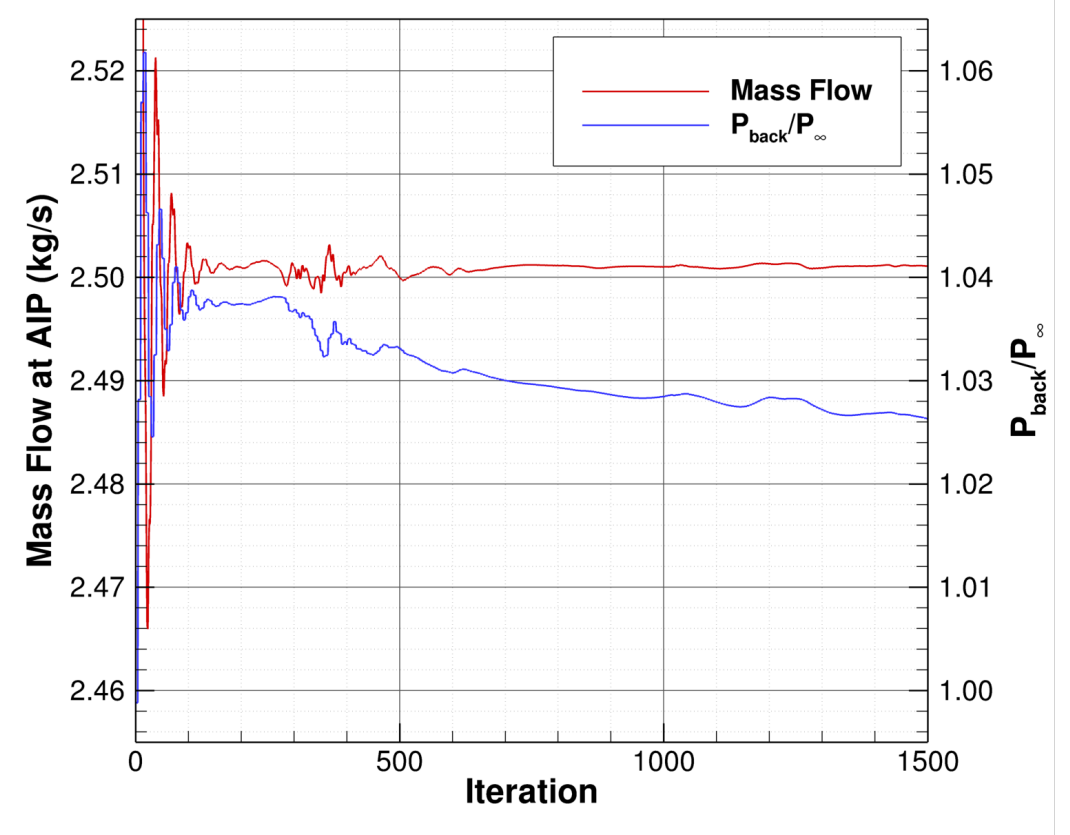

Fig. 22 Mass flow rate history for CFD2CFD case.

recovery numbers differ by less than $0.1 \%$, but the prediction of the distortion intensity is surprisingly large, greater than $20 \%$. Further study would be required to better understand the causes for the discrepancy. 

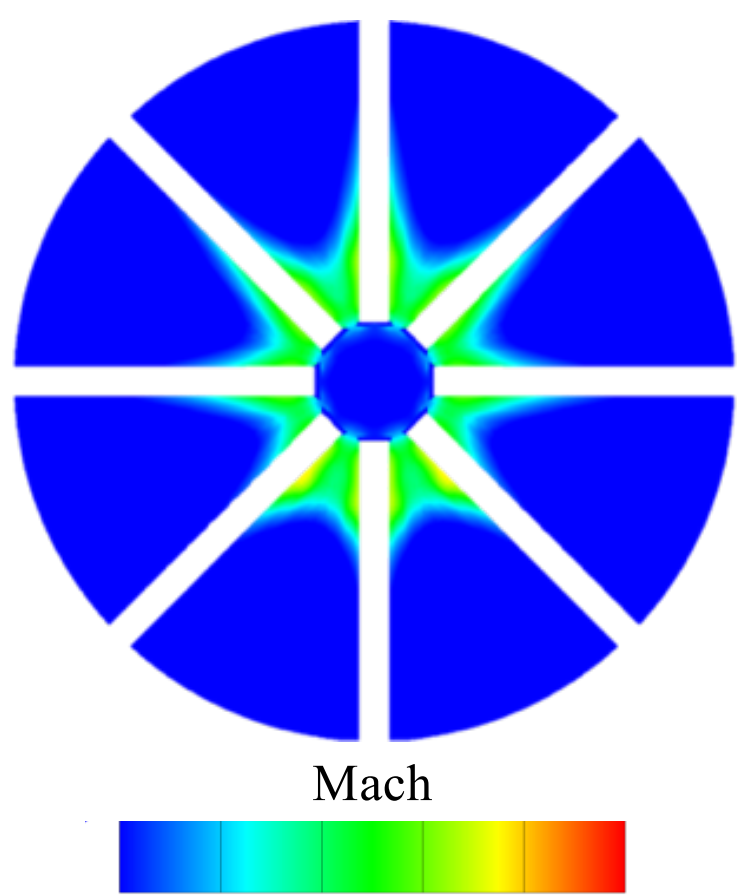

\section{$\begin{array}{llllll}0.9 & 1.0 & 1.1 & 1.2 & 1.3 & 1.4\end{array}$}

Fig. 23 Mach number contours at rake.

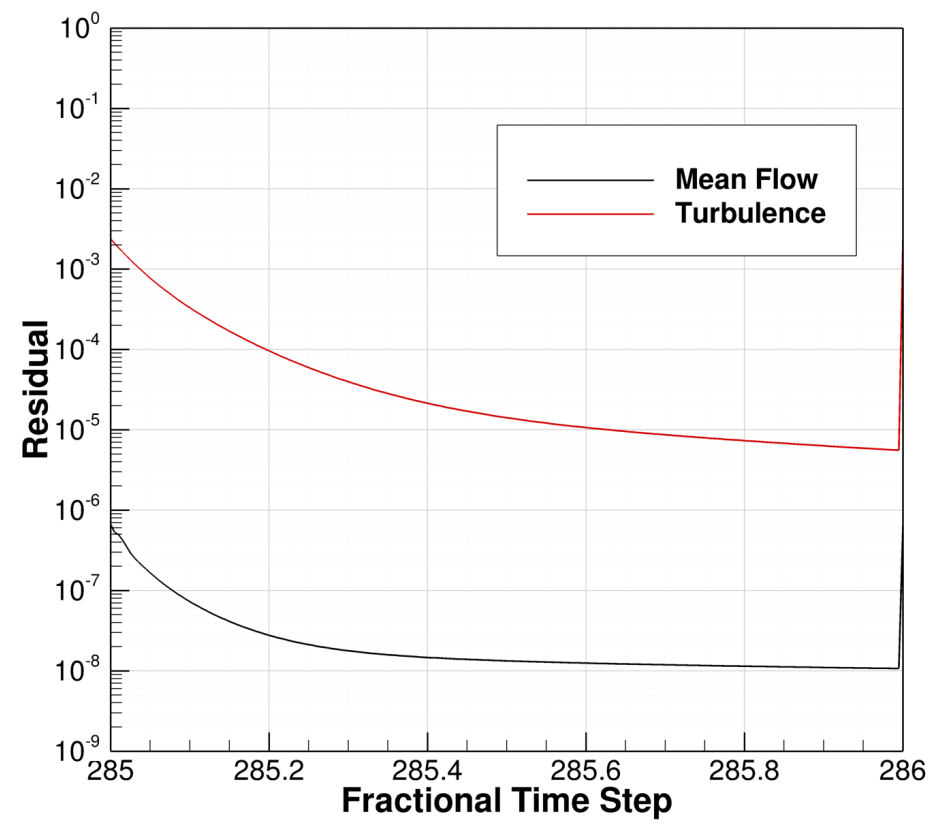

Fig. 24 Typical subiterative convergence for CFD2CFD simulations. 


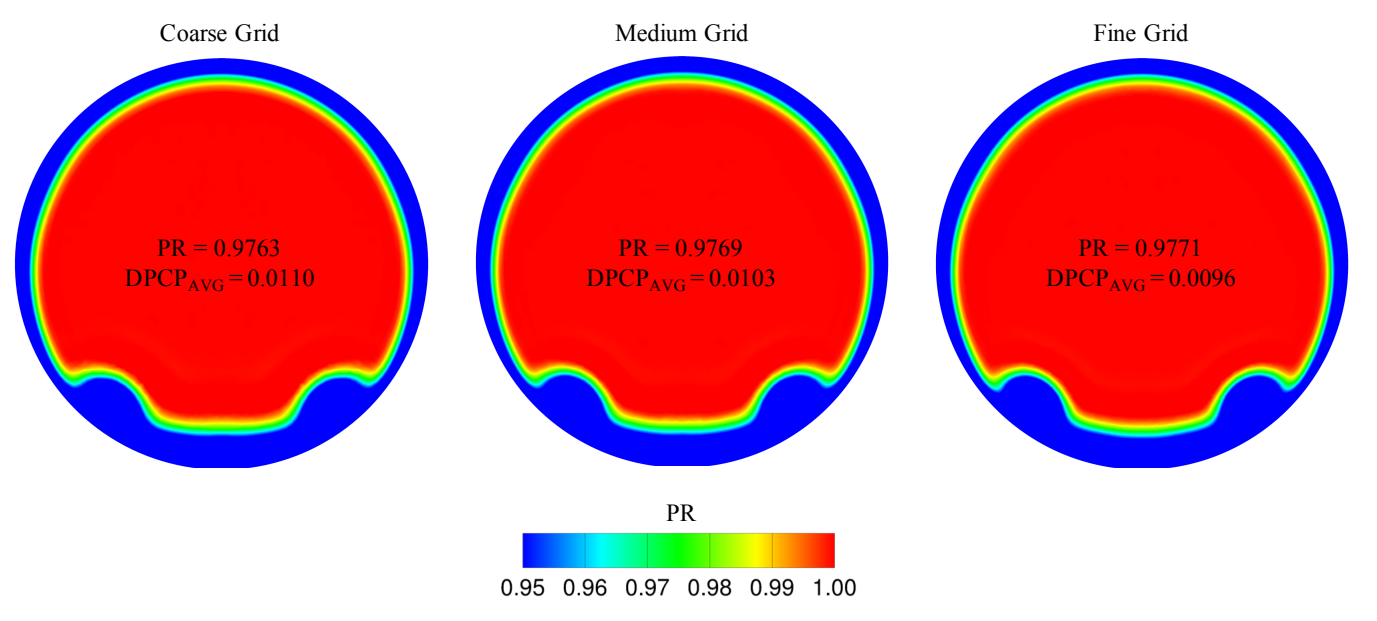

Fig. 25 Pressure recovery at AIP obtained from Fun3D for coarse, medium, and fine grids.

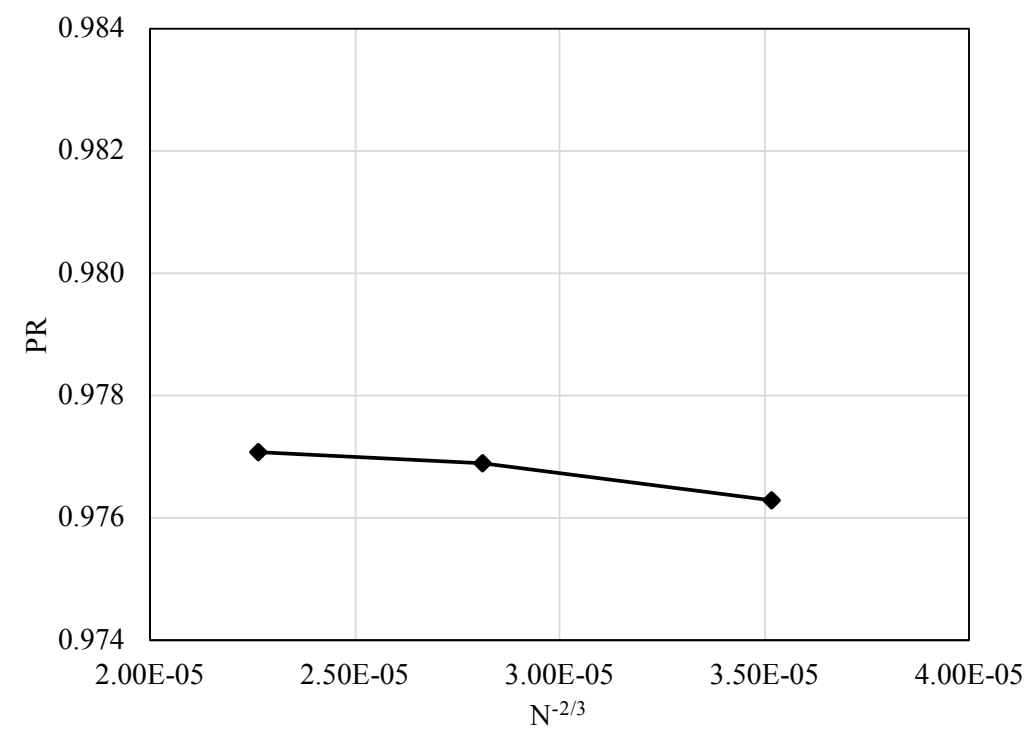

Fig. 26 Pressure recovery at AIP obtained from Fun3D as a function of grid refinement. 


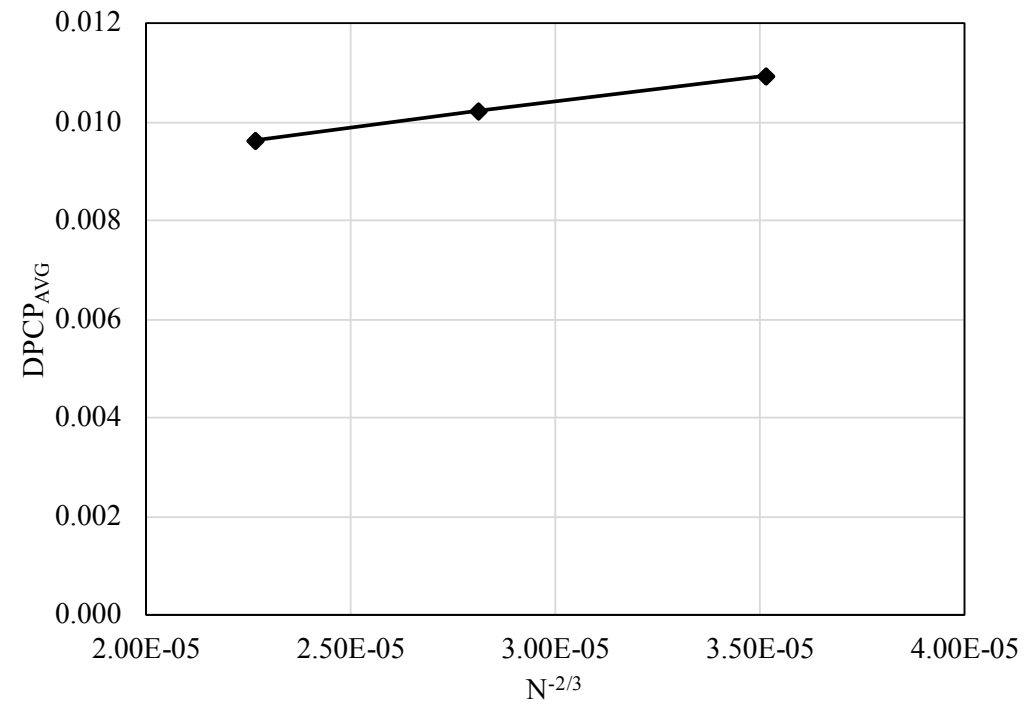

Fig. 27 DPCP at AIP obtained from Fun3D as a function of grid refinement.
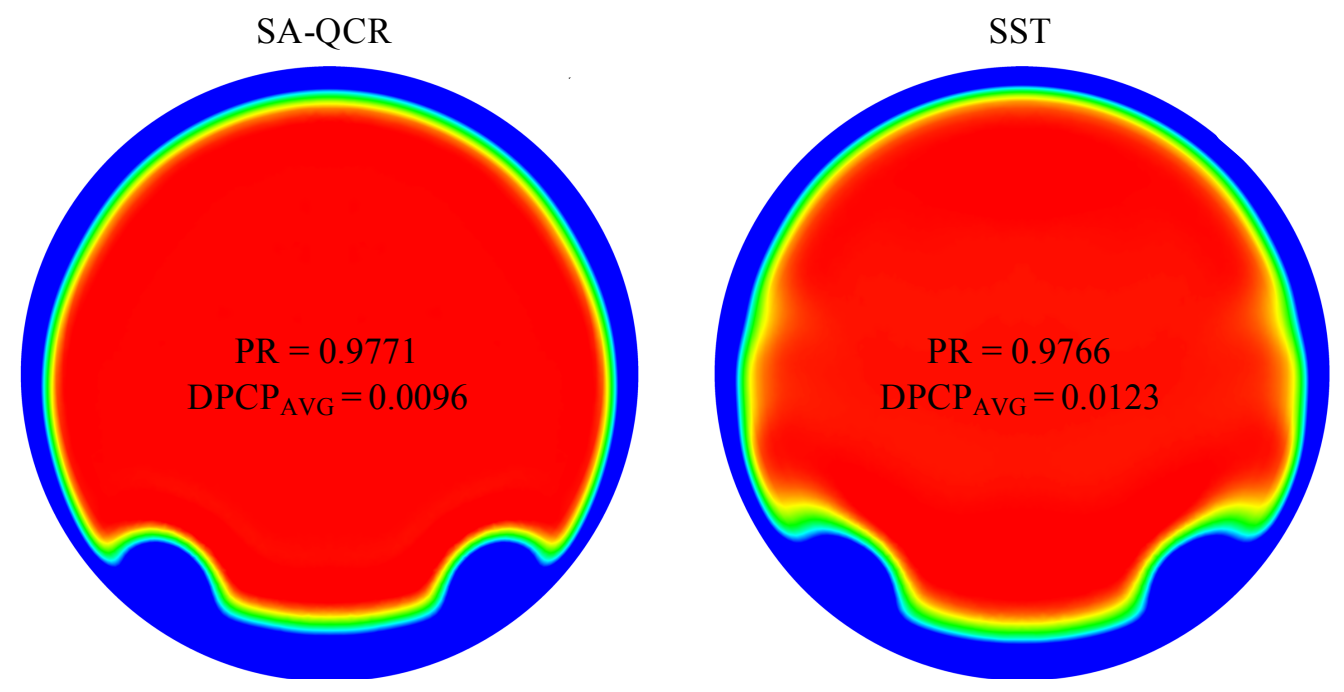

PR

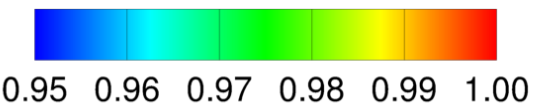

Fig. 28 Comparison of pressure recovery at AIP obtained from Fun3D using SA-QCR and SST turbulence models. 


\section{Summary}

This paper presented the FUn3D and USM3D analyses that were performed for the inlet configuration in participation of the 4th AIAA Propulsion Aerodynamics Workshop. The required analyses included a grid refinement study and turbulence model study for the two different comparison goals: CFD-to-CFD and CFD to experimental. Two additional studies, steady versus time-accurate and half- vs full-span simulations, were conducted. This work also included a grid generation exercise to accommodate the USM3D flow solver.

The grid refinement study illustrated very little differences between the predictions for the coarse, medium, and fine grids. This is likely due to the very close spacing in the grid resolutions, which is potentially an area of improvement for the future workshops. The CFD2EXP studies showed the success of an external and internal controllers for USM3D and Fun3D, respectively. However, for the CFD2CFD studies, the external controller for USM3D was unable to obtain the desired condition due to localized shocks that choked the flow around the AIP rakes. The steady versus time-accurate study showed that for Fun3D with the internal controller, time accurate without a flux limiter was best for predicting symmetrical flows. The half- versus full-span study showed that when Fun3D was run time-accurate without the limiter, a half-span grid was acceptable. The variation in pressure recovery with turbulence model was quite small for the cases investigated. Further study is require to understand the larger discrepancy in the predicted distortion intensities in the CFD2CFD comparisons.

When comparing the SST and SA-QCR turbulence models, it was seen that the persistence of cross-flow vortices were stronger with SST when compared to SA-QCR. Despite the nuance in the shapes of the flow distortion in the lower portion of the duct, the pressure recovery and distortion numbers are fairly close, with slightly over $0.5 \%$ variation in PR and $1 \%$ variation in distortion intensity.

Finally, all of the results were only off from the published experimental results at the thousandths value. Although this case has minimal distortion, this type of agreement is excellent in the opinion of these authors.

Future study possibilities include better integrating the controls with USM3D and running steady and time-accurate full span runs in an attempt to duplicate the results seen with Fun3D. Additionally, with a new version of USM3D being released shortly with mixed element capability, a reexamination of results between the two codes using similar grids should be conducted along with another grid resolution study in which the medium grid has 8 times as many points as the coarse grid $(2 \mathrm{x})(2 \mathrm{y})(2 \mathrm{z})$ and the fine grid has 64 times as many points $(4 \mathrm{x})(4 \mathrm{y})(4 \mathrm{z})$.

\section{Acknowledgments}

The authors would like to acknowledge the 4th Propulsion Aerodynamic Workshop committee members for their hard work organizing and conducting another successful workshop. Additionally, special thanks is given to Steve Krist, Farhad Ghaffari, and the late Khaled Abdol-Hamid, all from the NASA Langley Research Center for their guidance and long discussions regarding the unsteadiness of this problem. Finally, thanks to Patrick Shea who helped with the DPCP section writeup. 


\section{References}

[1] Delot, A.-L., and Scharnhorst, R., "A Comparison of Several CFD Codes with Experimental Data in a Diffusing S-duct," 49th AIAA/ASME/SAE/ASEE Joint Propulsion Conference, 2013, p. 3796.

[2] Bozeman, M. D., and Carter, M. B., "FUN3D and USM3D Analysis of the 4th AIAA Propulsion Aerodynamic Workshop Nozzle Test Case,” 2019 AIAA Propulsion \& Energy Forum, 2019.

[3] McMillan, M. L., Mackie, S. A., Gissen, A., Vukasinovic, B., Lakebrink, M. T., Glezer, A., Mani, M., and Mace, J. L., "Inlet Flow Control and Prediction Technologies for Embedded Propulsion Systems," NASA CR-2011-217237, December 2011.

[4] “Inlet Total-Pressure-Distortion Considerations for Gas-Turbine Engines,", SAE Aerospace Information Report 1419, 2013.

[5] Frink, N. T., "Tetrahedral Unstructured Navier-Stokes Method for Turbulent Flows," AIAA Journal, Vol. 36, No. 11, 1998, pp. $1975-1982$.

[6] Roe, P., "Characteristic-based schemes for the Euler equations," Annual review of fluid mechanics, Vol. 18, 1986, p. $337 ? 365$.

[7] Spalart, P. R., and Allmaras, S. R., “A One-Equation Turbulence Model for Aerodynamic Flows," La Recherche Aerospatiale, Vol. 1, No. 1, 1994, pp. 5-21.

[8] Spalart, P. R., "Strategies for Turbulence Modelling and Simulation," International Journal of Heat and Fluid Flow, Vol. 21, 2000, pp. 252-263.

[9] Menter, F. R., "Improved two-equation k-omega turbulence models for aerodynamic flows," NASA TM-103975, 1992.

[10] Roe, P. L., “Approximate Riemann Solvers, Parameter Vectors, and Difference Schemes,” J. Comp. Phys., Vol. 43, 1981, pp. $357-372$.

[11] van Leer, B., “Towards the Ultimate Conservative Difference Schemes V. A second order sequel to Godunov's Method,” J. Comp. Phys., Vol. 32, 1979, pp. 101-136.

[12] Shur, M. L., Strelets, M. K., Travin, A. K., and Spalart, P. R., "Turbulence Modeling in Rotating and Curved Channels: Assessing the Spalart-Shur Correction,” AIAA Journal, Vol. 38, No. 5, 2000, pp. 784-702. doi:10.2514/2.1058.

[13] Spalart, P. R., "Strategies for Turbulence Modelling and Simulations," International Journal of Heat and Fluid Flow, Vol. 21, No. 3, 2000, pp. 252-263. doi:10.1016/S0142-727X(00)00007-2.

[14] Biedron, R. T., Carlson, J.-R., Derlaga, J. M., Gnoffo, P. A., Hammond, D. P., Jones, W. T., Kleb, W., Lee-Rausch, E. M., Nielsen, E. J., and Park, M. A., “FUN3D Manual: 13.2,” NASA TM-2017-219661, August 2017.

[15] NASA Langley Research Center, "FUN3D," https://fun3d.larc.nasa.gov

[16] Jirasek, A., "Mass Flow Boundary Conditions for Subsonic Inflow and Outflow Boundary," AIAA Journal, Vol. 44, No. 5, 2006, pp. 939-947.

[17] Krist, S. L., Biedron, R. T., and Rumsey, C. L., “CFL3D User's Manual (Version 5.0),” NASA TM-1998-208444, June 1998.

[18] Carlson, J., "Inflow/Outflow Boundary Conditions with Application to FUN3D,” NASA TM-2011-217181, October 2011.

[19] Pointwise, R., "Release 18.2, Pointwise User Manual, Pointwise,”, 2018.

[20] Pandya, M. J., Diskin, B., Thomas, J. L., and Frink, N. T., "Assessment of USM3D Hierarchical Adaptive Nonlinear Method Preconditioners for Three-Dimensional Cases," AIAA Journal, 2017, pp. 3409-3424. 